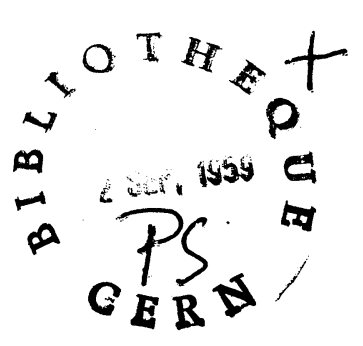

CERN LIBRARIES, GENEVA

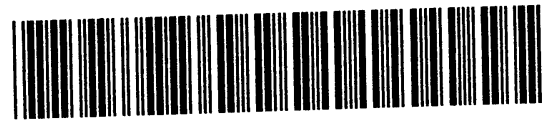

CM-P00066550

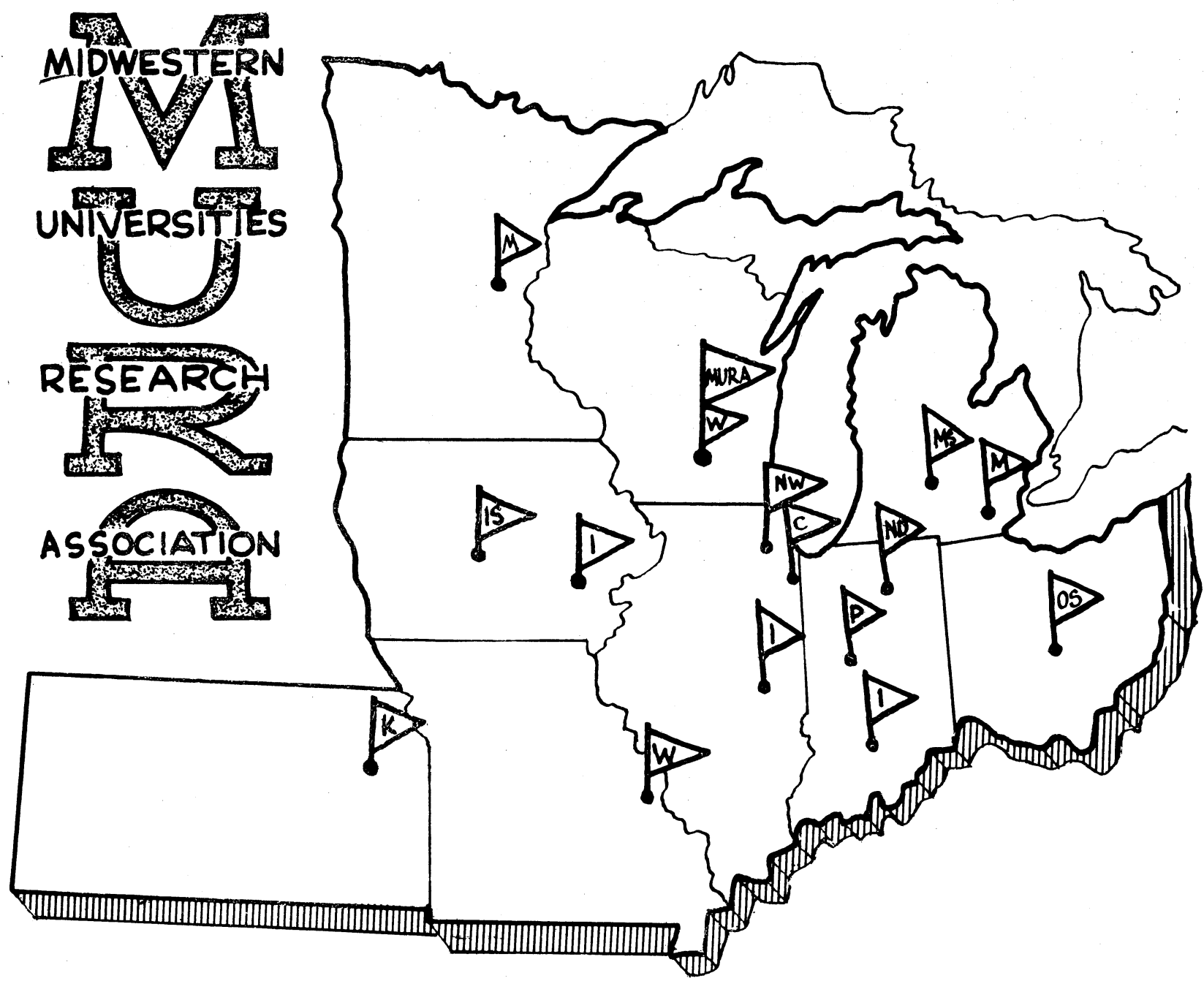

SETS OF EIGENVECTORS FOR VOLUMES OF REVOLUTION

J. Van Bladel

REPORT

a 537.212

b $538.12 \mathrm{~J}$ 
Printed in USA. Price \$1.00. Available from the

Office of Technical Services

U. S. Department of Commerce

Washington 25, D.C. 
MIDWESTERN UNIVERSITIES RESEARCH ASSOCIATION *

2203 University Avenue, Madison, Wisconsin

\section{SETS OF EIGENVECTORS FOR VOLUMES OF REVOLUTION}

J. Van Bladel $* *$

July 8, 1959

\section{ABSTRACT}

The electric and magnetic eigenvectors of a volume of revolution can be written in terms of two-dimensional scalar and vector functions. These functions are the eigenfunctions of certain linear transformations in the meridian plane. The form of the transformations is examined, and much attention is devoted to the orthogonality properties of their eigenfunctions and the relation of their eigenvalues to variational principles.

* AEC Research and Development Report. Research supported by the Atomic Energy Commission, Contract No. AEC AT(11-1)-384.

**Department of Electrical Engineering, University of Wisconsin, Madison, Wis. 


\section{TABLE OF CONTENTS}

\section{Preliminary remarks}

(a) Fields in volumes of revolution

(b) Electric eigenvectors

(c) Magnetic eigenvectors

(d) Variational principle for eigenvalues

II. Electric modes in toroidal volumes of revolution

(a) Irrotational eigenvectors

a. 1 Modes of revolution

a.2 Azimuth-dependent modes

(b) Solenoidal eigenvectors

b. 1 Modes of revolution

1. Circular modes.

2. Meridian modes

b. 2 Azimuth-dependent modes

III. Expansion in electric eigenvectors

IV. Magnetic modes in toroidal volumes of revolution

(a) Irrotational eigenvectors

$a_{\circ} 1$ Modes of revolution

a. 2 Azimuth-dependent modes

(b) Solenoidal eigenvectors

b. 1 Modes of revolution

1. Circular modes

2. Meridian modes

b. 2 Azimuth-dependent modes

V. Expansion in magnetic eigenvectors

VI. Regions containing the axis 
MURA-481

TABLE OF CONTENTS (continued)

VII. Application to the circular cylindrical cavity.

Appendices

1. Differential operators in a meridian plane.

2. Properties of the operator $\mathcal{L}=\nabla_{M}^{2}-\frac{m^{2}}{\Omega^{2}}+\frac{2}{r} \bar{u}_{\Omega} d_{M}$

3. Behavior of a continuous vector in the vicinity of the axis.

3 
Among the sets of eigenvectors which exist in a finite three-dimensional volume the "electric" and "magnetic" modes 1 are of particular importance for the calculation of electric and magnetic fields. The purpose of the present paper is to investigate the properties of these modes in volumes of revolution of the kind depicted in Fig. 1. An explicit mathematical expression can be given for the modes of a few simple volumes, such as the sphere and the coaxial cylinder, but in the most general case one has to resort to approximate procedures to obtain guantitative data. The most frequently used methods rely on the replacement of differential equations by difference equations, and on the use of variational principles for the calculation of eigervalues. It is necessary, for a systematic appilication of the se methods, to possess a precise classification and enumeration of the modes and their characteristics. This is what these pages, inspired by a previous analysis by Bernier $r_{2}^{2}$ set out to provide. The treatment is quite general and, by the same token, somewhat unpleasantly dry。 In an effort to enliven the presentation, an application of the general theory to a particular cavity has been included.

The first structure to be examined will be the toroidal volume of Fig. 1a, which is of importance for circular particle accelerators and, more generally, for ring-like structures through which particles or fluids are flowing. The fact that a toroidal volume does not contain any portion of the axis of revolution facilitates the mathematical formulation of the problem.

1. For general background material, see MURA $-440_{2}$ "On Helmholtz's Theorem in Finite Regions" "December 1958\%

2. Jo Bernier。"On Electromagnetic Resonators", Onde Electrique \& 26 , pp。305-317, 1946。 

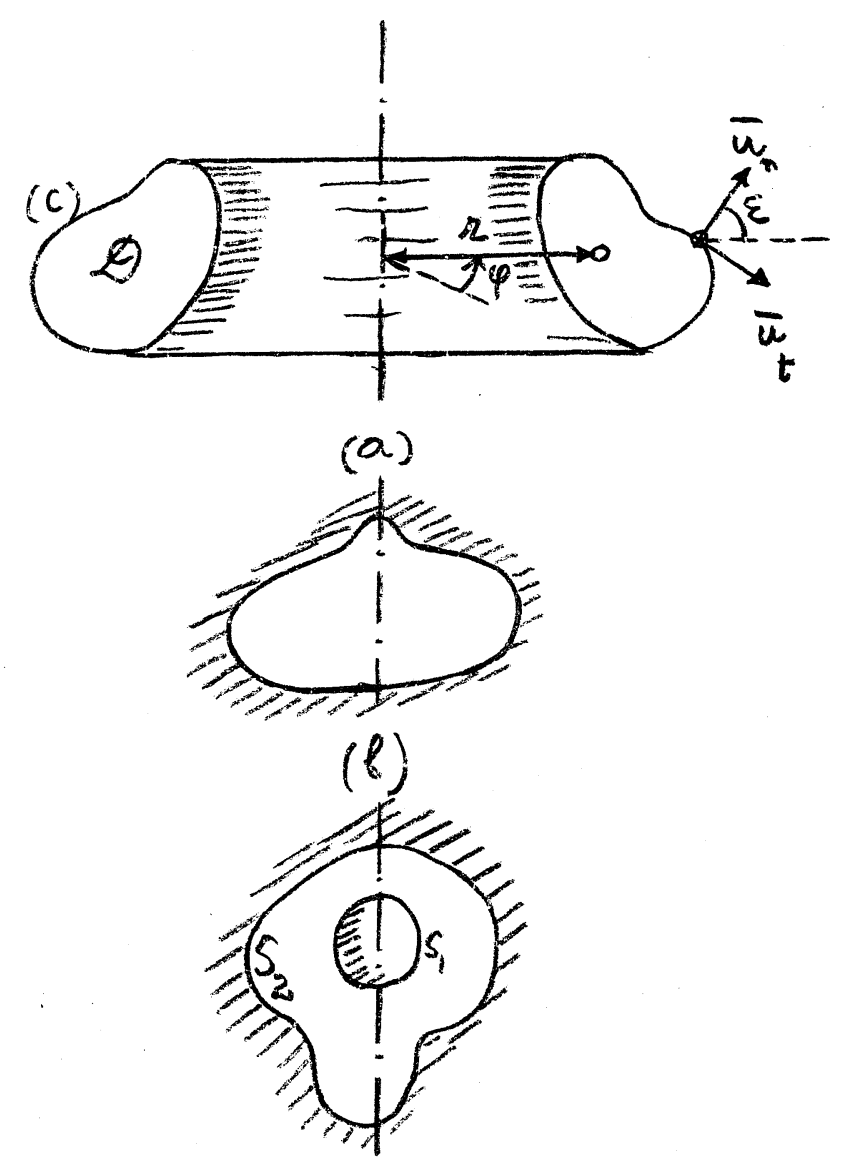

(c)

Fig。 1

\section{PRELIMINARY REMARKS}

(a) Fields in volumes of revolution

One of the problems to be investigated is the determination of the expansion coefficients of a piecewise continuous vector function $\bar{a}(r, z, \varphi)$. This determination is simplified by a preliminary Fourier expansion of $\bar{a}$ in sines and cosines.

$$
\begin{gathered}
\bar{a}(r, \xi, \varphi)=\bar{p}_{0}\left(r_{0} z\right)+v_{0}(r, z) \bar{u}_{\varphi}+\sum_{m} \sin m \varphi \cdot \bar{p}_{m}(r, z) \\
+\cos m \varphi \bar{q}_{m}(r, z)+\sum_{m}\left[-w_{m}(r, \xi) \sin m \varphi\right. \\
\left.+v_{m}\left(r_{0}, z\right) \cos m \varphi\right] \bar{u}_{\varphi}
\end{gathered}
$$


The $\overline{p^{i}} \mathrm{~s}$ and $\overline{\mathrm{q}^{9} \mathrm{~s}}$ are "meridian" vectors。 (i。e vectors situated in the meridian plane). Vectors such as $v_{0} \bar{u}_{\varphi}$, where $\bar{u}_{\varphi}$ is a unit vector perpendicular to the meridian plane and directed toward increasing $\varphi$, form the "circular" components. The divergence and curl of $\bar{a}$ are given by

$$
\begin{aligned}
& \operatorname{div} \overline{\mathrm{a}}=\operatorname{div}_{M} \overline{\mathrm{p}}_{\mathrm{o}}+\sum_{\mathrm{m}} \sin m \varphi\left(\operatorname{div}_{M} \overline{\mathrm{p}}_{\mathrm{m}}-\frac{m v_{m}}{\mathrm{r}}\right) \\
& +\sum_{m} \cos m \varphi\left(\operatorname{div}_{\mu} \bar{q}_{m}-\frac{m W_{m}}{r}\right) \\
& \operatorname{curl} \overline{\mathrm{a}}=\operatorname{curl} \overline{\mathrm{p}}_{\mathrm{O}}+\operatorname{curl}\left(\mathrm{v}_{\mathrm{O}} \bar{u}_{\varphi}\right)+\sum_{\mathrm{m}} \sin \mathrm{m} \varphi\left[\operatorname { c u r l } _ { \mathcal { M } } \mathrm { p } _ { \mathrm { m } } ^ { - \operatorname { c u r l } } { } _ { \mathcal { M } } \left(w_{m} \bar{u}_{\varphi}\right.\right. \\
& \left.-\frac{m}{r}\left(\bar{u}_{\varphi} \times \bar{q}_{m}\right)\right] \\
& +\sum_{m} \cos m \varphi\left[\operatorname{curl} \bar{q}_{M}+\operatorname{curl}\left(v_{M} \bar{u}_{\varphi}\right)+\frac{m}{r}\left(\bar{u}_{\varphi} \times \bar{p}_{m}\right)\right] \text {. }
\end{aligned}
$$

Differential operators having the subscript $M$ ( $\mathcal{M}$ for meridian) are defined in Appendix I. When $\bar{a}$ is solenoidal (i。e, $\operatorname{div} \bar{a}=0$ ), the following relations hold:

$$
\operatorname{div}_{M} \bar{p}_{\mathrm{O}}=0 \quad \operatorname{div}_{\mathcal{M}} \overline{\mathrm{p}}_{\mathrm{m}}=\frac{m v_{m}}{\mathrm{r}} \quad \operatorname{div}_{\mathcal{M}} \overline{\mathrm{q}}_{\mathrm{m}}=\frac{m w_{m}}{\mathrm{r}}
$$

When $\bar{a}$ is irrotational (i。e curl $\bar{a}=0$ )

$$
\begin{aligned}
& \operatorname{curl}{ }_{M} \vec{p}_{0}=\operatorname{curl}_{M} \bar{p}_{m}=\operatorname{curl}_{M} \bar{q}_{m}=\operatorname{curl}_{\mathcal{M}}\left(v_{O} \bar{u}_{\varphi}\right)=0 \\
& \operatorname{corrl}{ }_{M}\left(v_{m} \bar{u}_{\varphi}\right)=-\frac{m}{r}\left(\bar{u}_{\varphi} \times \bar{p}_{m}\right) \\
& \operatorname{curl}_{M}\left(w_{m} \bar{u}_{\varphi}\right)=-\frac{m}{r}\left(\bar{u}_{\varphi} \times \bar{q}_{m}\right)
\end{aligned}
$$

\section{(b) Electrice eigenvectors}

The electric eigenvectors of a simply-bounded volume fall into two categories: 
-Irrotational eigenvectors $\bar{f}_{m n p}=\operatorname{grad} \psi_{m n p}$ where $\psi_{m n p}$ is an eigenfunction of the problem

$$
\nabla^{2} \psi_{m n p}+\lambda_{m n p}^{\prime} \psi_{m n p}=0 \quad \psi_{m n p}=0 \text { on boundary surface s }
$$

The triple index accounts for the triple infinity of eigenfunctions.

-Solenoidal eigenvectors $\overline{\mathrm{e}}_{\mathrm{mn}}$ p solutions of

$$
- \text { curl curl } \overline{\mathrm{e}}_{\mathrm{mnp}}+\lambda_{\mathrm{mnp}}^{\prime \prime} \overline{\mathrm{e}}_{\mathrm{mnp}}=0 \quad \bar{u}_{m} \times \overline{\mathrm{e}}_{\mathrm{mnp}}=0 \text { on boundary surface } \mathrm{s} r^{\prime}
$$

The notation $\bar{u}$ stands for "unit vector", and $\bar{u}_{n}$ is the unit vector along the outward-pointing normal to S.

(c) Magnetic eigenvectors

The complete set of magnetic eigenvectors of a toroidal volume consists of

-A single "sourceless" vector $\overline{\mathrm{h}}_{\mathrm{O}}=\operatorname{grad} \theta_{\mathrm{O}}$, tangent to the boundary surface。*

-Irrotational eigenvectors $\bar{g}_{\mathrm{mnp}}=\operatorname{grad} \theta_{\mathrm{mnp}}$ where $\theta_{\mathrm{mnp}}$ is an eigenfunction of the problem.

$$
\begin{aligned}
& \nabla_{m n p}^{2}+\varphi_{m i n} \theta_{m n p}=0 \quad \frac{\partial \theta_{m n p}}{\partial n}=0 \text { on } \mathrm{S} \\
& \text {-Solenoidal eigenvectors } \bar{h}_{m n p} \text { solutions of } \\
& \text {-curl curl } h_{m n p}+\mu_{m n p} \bar{h}_{m n p}=0 \quad \bar{u}_{m} \times \operatorname{curl} \bar{h}_{m n p}=0 \text { on } \mathrm{S} \text {. }
\end{aligned}
$$

It can be shown that the eigenvalues $\mu$ and $\lambda^{\prime \prime}$ are identical, and that the electric and magnetic solenoidal eigenvectors are multiples of the curl of each other. In other words, $\overline{\mathrm{e}}_{\mathrm{mnp}}$ is proportional to curl $\overline{\mathrm{h}}_{\mathrm{mnp}}$ and $\overline{\mathrm{h}}_{\mathrm{mnp}}$ is

\footnotetext{
*We define a "sourceless" vector as having zero divergence and zero curl.
} 
proportional to curl $\overline{\mathrm{e}}_{\mathrm{mnp}}$. The proportionality constants depend on the normalization of the eigenvectors.

(d) Variational principle for eigenvalues

Variational properties are of considerable interest for the approximate determination of eigenvalues and eigenvectors when the boundaries are irregular in shape. The basic property is as follows: When $\mathcal{L}$ is a negative definite self-adjoint transformation, all eigenvalues in $\mathcal{L} u_{m}+\lambda_{m} u_{m}=0$ are real and positive. Denoting by $\langle a, b\rangle$ the scalar product of a by $b$, the lowest eigenvalue $\lambda_{1}$ is the minimum of $J(u)=-\frac{\langle\mathcal{L} u, u\rangle}{\langle u, u\rangle}$. This minimum is attained for the lowest eigenfunction $u_{1}$. The functions admitted for competition (the "admissible" functions) must belong to the space of definition of the transformation $\mathcal{L}$. The second lowest eigenvalue is the minimum value of $J$ with respect to admissible functions that are orthogonal to $u_{1}$, (i.e. for which $\left\langle u, u_{1}\right\rangle=0$ ), and the minimum is attained for $u=u_{2}$. Similarly, $\lambda_{m}$ is the minimum of $J$ with respect to $u^{\prime}$ 's that are orthogonal to the $(n-1)$ lowest eigenfunctions, and the minimum is attained for $u=u_{n}$. Similar results are obtained, mutatis mutandis, for positive-definite transformations.

These considerations can be applied to transformation (6) and (8). The scalar product to be used here is $\iiint_{V} a b d V$, and the $\lambda_{\text {mnp }}$ are obtained as stationary values of the expression

$$
J(\psi)=-\frac{\iiint_{V} \psi \nabla^{2} \psi d V}{\iiint_{V} \psi^{2} d V}
$$

The admissible functions vanish on the boundary, and are continuous up to their

* $\mathcal{L}$ is self-adjoint when $\langle u, \mathcal{L} v\rangle=\langle\mathcal{L} u, v\rangle$ for all $u, v$ belonging to the space of definition of $\mathcal{L}$, and it is negative definite when $\langle u, \mathcal{L} u\rangle \leq 0$, the equality sign being obtained for, and only for, $u=0$. These properties are associated with a specific definition of the scalar product 
second derivatyes. The eigenvalues $V_{\text {mnp }}$ are obtained as stationary values of the same expression, the admissible functions having the same continuity properties, but a vanishing normal derivative on $\mathrm{S}_{0}$

Transformation (7) with scalar product $\langle\bar{a}, \bar{b}\rangle=\iiint_{V} \bar{a} \cdot \bar{b} d V$ Ieads to the characterization of $\lambda_{\mathrm{mnp}}^{\prime \prime}$ as stationary value of the expression

$$
J(\bar{e})=\frac{\iint_{V} \bar{e} \cdot \operatorname{cunt} \operatorname{cusl} \bar{e} d V}{\iint_{V} \bar{e} \cdot \bar{e} d V}
$$

where the admissible vectors have zero divergence, are continuous up to their second derivatives and are perpendicular to the boundary surface.

\section{ELECTRIC MODES IN TOROIDAL VOLUMES OF REVOLUTION}

The general considerations of the preceding paragraph will now be applied more specifically to volumes of revolution. In each category a distinction will be made between modes of revolution ( the meridian and circular components of which are independent of azimuth $\varphi$ ) and $\varphi$-dependent modes。

\section{(a) Irrotational eigenvectors}

\section{a. 1 IModes of revolution}

The $\varphi$ independent modes $\bar{f}_{\text {onp }}$ are gradients of the eigenfunctions

of

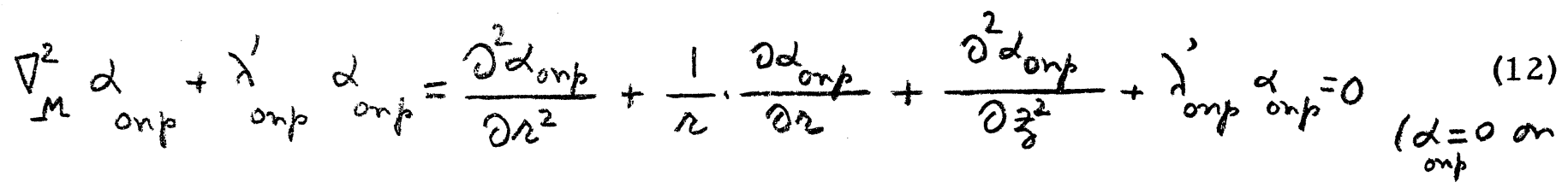

The problem is selfadjoint and negative definite with respect to a scalar product $\langle a, b\rangle=\iint_{\partial} a b r d r d z$. The various eigenfunctions are orthogonal in the

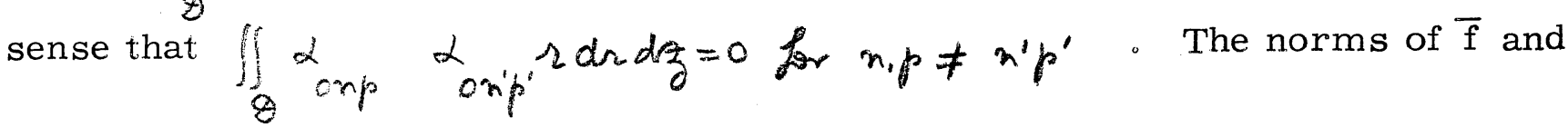


$\alpha \quad$ are related by the formula

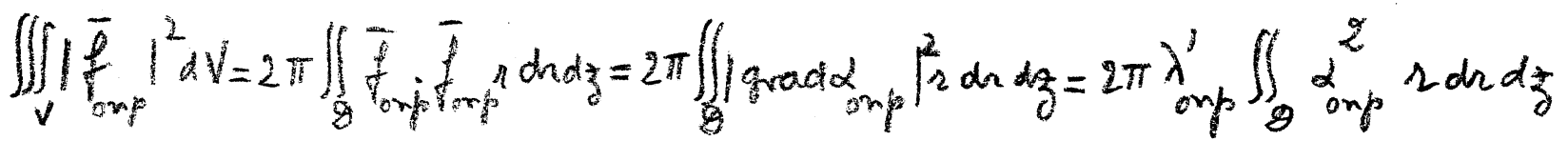

obtained by an application of (A1, 16)。* The eigenvalues can be obtained as stationary values of

$$
J(\alpha)=-\frac{\iint_{\theta} \alpha \nabla_{\alpha}^{2} \alpha r d r d z}{\iint_{\theta} \alpha^{2} r d r d z}
$$

The admissible functions vanish on boundary (c) and have continuous derivatives up to the second order.

\section{a. 2 Azimuth-dependent modes}

The $\varphi$ dependent modes are periodic in $\varphi$, and their

expression is

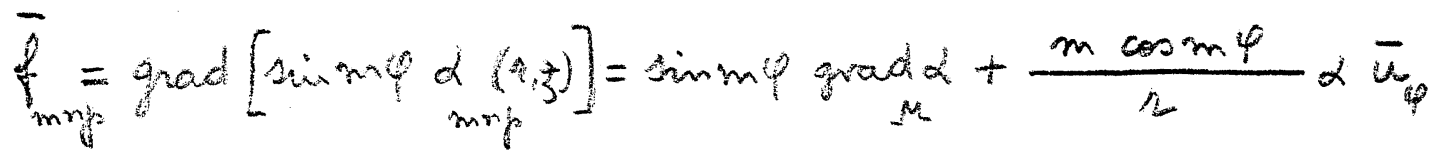

The functions $\alpha$ are eigenfunctions of the problem

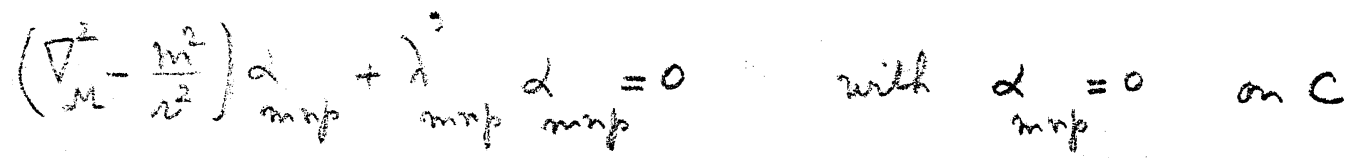

The usual $\varphi$-degeneracy is encountered, $i_{0} e_{0}$ two modes, $\operatorname{grad}[\sin m \varphi \alpha]$ and grad $[\cos m \alpha]$, correspond to each value of $\lambda^{3}$. This characteristic property will be found for all other 4 -dependent modes to be examined in the future. For reasons of conciseness, one of the modes only will be written down explicitly. The second one can then be obtained by simply increasing $m \varphi$ by $\pi / 2$.

The problem defined by (15) is again self-adjoint and negative definite with respect to the scalar product $\int_{\theta} a b d n d z$. The $\alpha_{\text {mnp }}$ are orthogonal

\footnotetext{
*The formulas in the appendices are identified by a prefix A.
} 


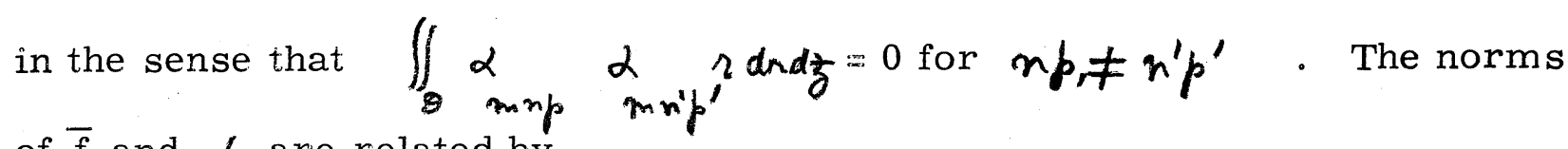
of $\bar{f}$ and $\alpha$ are related by

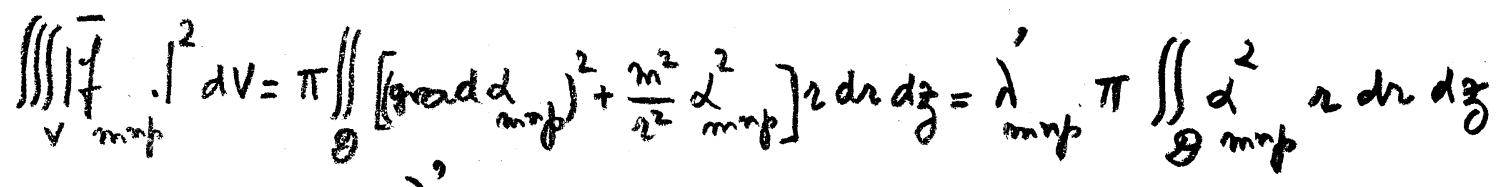
The eigenvalues $\lambda_{\text {mnp }}$ can be obtained as stationary values of

$$
J(\alpha)=-\frac{\iint_{\theta} \alpha\left[\nabla_{M}^{2} \alpha-\frac{m^{2} \alpha}{r^{2}}\right] r d r d z}{\iint_{g} \alpha^{2} r d r d z}
$$

the admissible functions being the same as in (14).

(b) Solenoidal eigenvectors

b. 1 Modes of revolution

The solenoidal eigenvectors $\overline{\mathrm{e}}_{\text {onp }}$ can usefully be split into a meridian and a circular part according to the formula

$$
\overrightarrow{\mathrm{e}}_{\text {onp }}=\overline{\mathrm{c}}_{\text {onp }}(\mathrm{r}, z)+\beta_{\text {onp }}(r, z) \bar{u}_{\varphi}
$$

If the latter expression is plugged into (7), and the $\varphi$-independence taken into account, uncoupled equations are obtained for $\bar{c}$ and $\beta$. The modes are consequently of two different sorts。

\section{1) Circular modes $\beta$ onp $\bar{\psi}$}

There is a double infinity of these modes, corresponding to the eigenfunctions of the problem

$$
\nabla_{m}^{2} \beta_{\text {onp }}-\frac{\beta_{\text {ond }}}{r^{2}}+\lambda_{\text {onp }}^{\prime \prime} \beta_{\text {onp }}=0 \quad \beta_{\text {onp }}=0 \text { on } C
$$


The $\beta_{\text {onp }}$ are, in consequence, equal to the functions $\alpha_{\text {inp }}$ encountered in paragraph $\left(a_{0} 2\right)$, and partake of their orthogonality and stationarity properties. The eigenvalues $\lambda_{\text {oxp }}^{\prime \prime}$ of the circular modes are equal to the $\lambda_{\text {'mp }}^{\prime}$. The normalization is particularly simple:

2) Meridian modes $\bar{C}_{\text {onp }}$

$$
\iiint_{V} \beta \bar{u}_{\varphi} \cdot \beta \bar{u}_{\varphi} d V=2 \pi \iint_{\theta} \beta^{2} r d r d z
$$

The eigenvalue problem satisfied by the $\bar{C}_{\text {orp }}$ is

- curl curl $\bar{C}_{\text {onp }}+\lambda_{\text {onp }}^{\prime \prime} \bar{c}_{\text {onp }}=0 \quad$ with $\left\{\begin{array}{l}\bar{u}_{m} \times \bar{c}_{o n p}=0 \\ \operatorname{diw}_{M} \bar{C}_{o n p}=0\end{array}\right.$ on (C)

It is a simple matter to show that the se meridian vectors are actually the curl of the circular magnetic eigenvectors. More precisely, the $\bar{C}_{\text {onp }}$ can be put in the form

$\operatorname{cunl}\left[\delta_{\text {onp }} \bar{u}_{\varphi}\right]=-\frac{\partial \delta_{\text {onp }}}{\partial z} \bar{u}_{r}+\left(\frac{\partial \delta_{\text {onp }}}{\partial r}+\frac{\delta_{\text {onp }}}{r}\right) \bar{u}_{z}=\frac{1}{r}\left[\operatorname{grad}\left(\delta_{o n p} r\right) \times \bar{u}_{\varphi}\right]$

where the functions $\delta_{\text {orp }}$ satisfy the eigenvalue problem

$$
\left(\nabla_{\mu}^{2}-\frac{1}{r^{2}}\right) \delta_{o n p}+\lambda_{o n p}^{\prime \prime} \delta_{o n p}=0 \text { with } \bar{u} \times \operatorname{aul}\left[\delta_{o_{p p}} \bar{u}_{\varphi}\right]=0 \text { on }(C)
$$

The boundary condition can be rewritten in the form

$$
\frac{1}{r} \frac{\partial}{\partial n}\left(\delta_{o n p} r\right)=\frac{\partial \delta_{o n p}}{\partial n}+\frac{\delta_{o n p}}{r} \cdot \cos \varepsilon=0
$$

The $\delta_{\text {onp }}$ are orthogonal with respect to a scalar product $\iint_{\theta} a b \Omega d \Omega d z$ and the eigenvalues are the stationary values of

$$
J(\delta)=-\frac{\iint_{\theta} \delta\left(\nabla_{M}^{2} \delta-\frac{\delta}{n^{2}}\right) r d r d z}{\iint_{\theta} \delta^{2} r d r d z}
$$

The admissible functions are required to possess the usual continuity properties, 
and to satisfy (24) at the boundary. ${ }^{3}$ The normalization integral is simply

$$
\iiint_{V} \bar{C}{ }_{\text {orp }} \cdot \bar{c} \text { oxp }^{\prime \prime} d V=2 \pi \lambda_{\text {onp }} \iint_{\theta} \delta_{o n p}^{2} r d r d z
$$

b. 2 Azimuth-dependent modes

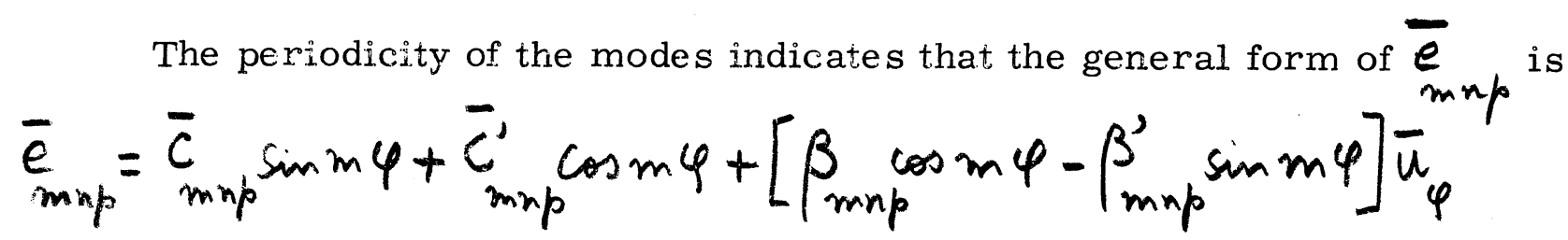

If the latter expression is inserted in (7), uncoupled and identical equations are obtained for the pairs $(\bar{c}, \beta)$ and $\left(\overline{c^{\prime}}, \beta^{\prime}\right)$. This fact indicates the existence of an eigenvector $\bar{c}_{m x p} \sin m \varphi+\beta_{m x p} \cos m \varphi \bar{u}_{\varphi}$, and also of an eigenvector $\left(\bar{c}^{\prime},-\beta^{\prime}\right)$ obtairied from the former by increasing $m \varphi$ by $\pi / 2$, i.e., by rotating the configuration through an angle $\frac{\pi}{2 m}$. The equations which $\underset{\mathrm{c} m \boldsymbol{p}}{\overline{\mathrm{m}}}$ and $\beta_{\text {mnp }}$ are required to satisfy are rather complicated. Dropping the subscripts for a moment, they turn out to be

$$
\begin{aligned}
& -\operatorname{arl} \operatorname{cunl}_{M} \bar{c}-\frac{m^{2} \bar{c}}{r^{2}}+\frac{m}{r} \operatorname{grad} \beta+\frac{m \beta}{r^{2}} \bar{u}_{r}+\lambda^{\prime \prime} \bar{c}=0 \\
& \nabla_{M}^{2} \beta-\frac{\beta}{r^{2}}+\frac{2 m}{r^{2}}\left(\bar{c} \cdot \bar{u}_{r}\right)-\frac{m}{r} \operatorname{diw}_{M} \bar{c}+\lambda^{\prime \prime} \beta=0
\end{aligned}
$$

The se equations can be simplified by taking into account the fact that $\bar{e}$ is solenoidal, $i$ 。 。 that

$$
\operatorname{di\omega }\left[\bar{c} \sin m \varphi+\beta \cos m \varphi \bar{u}_{\varphi}\right]=\sin m \varphi\left[\operatorname{diw}_{M} \bar{c}-\frac{m \beta}{r}\right]=0
$$

There exists, in consequence, a relation between $\beta$ and $\bar{C}$, namely ${ }^{*}$

$$
\beta=\frac{r}{m} \operatorname{div}_{\mu} \bar{c}
$$

3. For an example of calculation of $\beta_{o x p}$ and $\delta_{o n p}$ by difference-equation and variational methods, see D. F. Meronek and J。 Van Bladel "Resonant Modes and Frequencies of a Cigar-shaped Cavity". Microwave Journal, 2 pp. 32-33, 1959.

* Equation (29) can be obtained directly from (27) and (28) by computing the divergence of $(27)$, multiplying it by $\frac{n}{m}$, and comparing the resulting equation 
Upon substitution of this expression in (27), an equation for $\bar{C}$ alone is obtained.

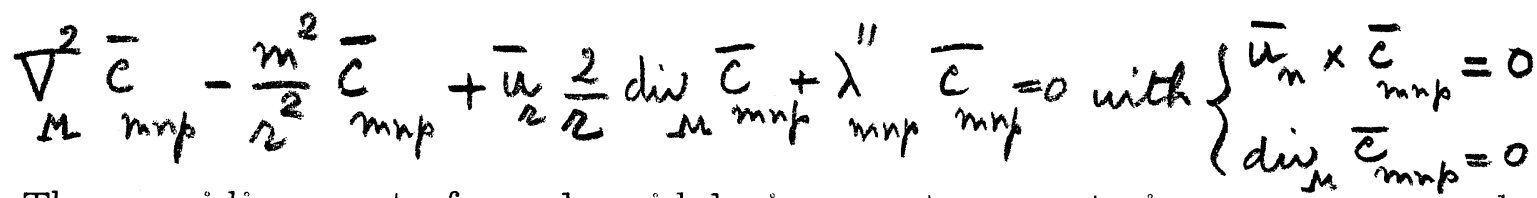

The meridian part of a sclenoidal eigenvector must, in consequence, be an

eigenvector of problem (30). Conversely, to each eigenvector of (30) corresponds an eigenvector

$$
\bar{c}_{\min p} \sin m \varphi+\frac{2}{m} \cdot \operatorname{div}_{\mu} \bar{c} \cdot \cos m \varphi \cdot \bar{u}_{\varphi}
$$

of the original three-dimensional problem (7), It is important to list orthogonality and stationarity properties of the $\bar{C}_{\text {map }}$. These properties can be obtained from the general equation (11) wherein (31) is substituted. They can also be established directly from a study of the transformation

$$
L \bar{v}=\nabla_{M}^{2} \bar{v}-\frac{m^{2}}{r^{2}} \bar{v}+\frac{2 \bar{u}_{n}}{2} d i v \bar{v} \quad \text { with }\left\{\begin{array}{l}
\bar{u}_{n} \times \bar{v}=0 \\
\operatorname{diw}_{M} \bar{v}=0
\end{array}\right. \text { on (c) }
$$

in the meridian plane. The relevant steps are collected in Appendix II. It turns out that the scalar product which is suited to the problem is

$\langle\bar{v}, \bar{N}\rangle=\iint_{\theta}\left[\bar{v} \cdot \bar{w}+\frac{n^{2}}{m^{2}} \operatorname{diw}_{M} \bar{v} \cdot d \operatorname{lin}_{M} \bar{w}\right] r d r d z$

where $\bar{v}$ and $\bar{W}$ are two meridian vectors. With the latter definition of the scalar product, transformation $\mathcal{L}$ is self-adjoint and negative-definite, the eigenvectors are orthogonal in the sense that

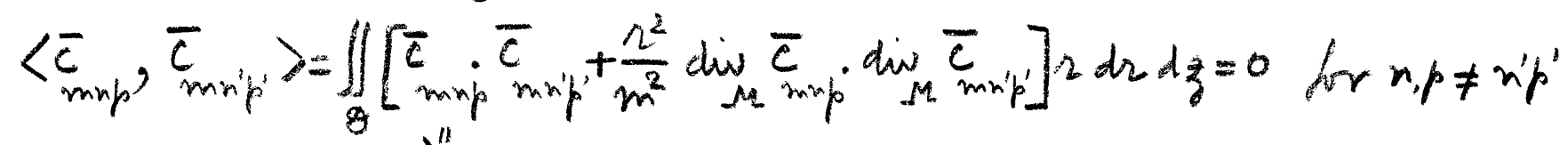
and the eigenvalues $\lambda^{n}$ mar are obtained from the stationarity properties of

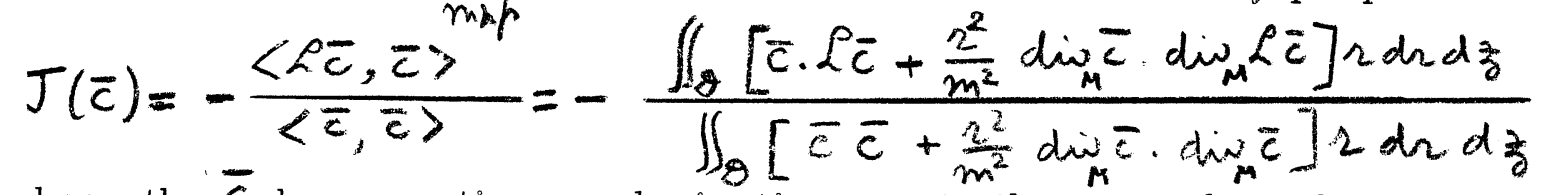

where the $\bar{C}$ have continuous derivatives up to the second, and satisfy the boundary conditions evidenced in (32). Third order derivatives appear in the numerator. An equivalent expression for the latter can be derived which involves 
lesser order derivatives only. The derivation is based on the relation

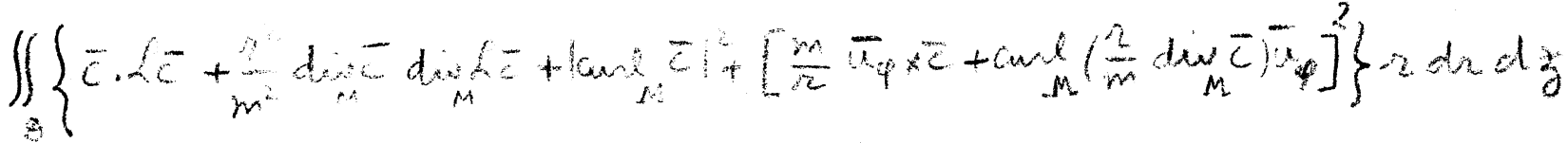

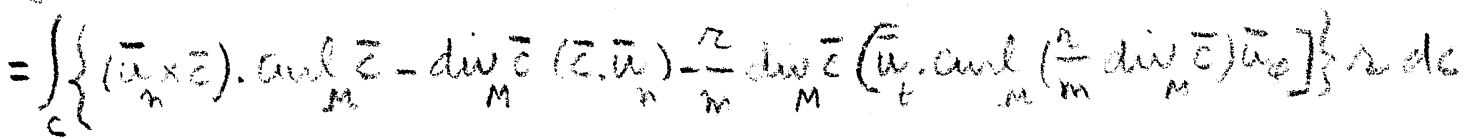

a direct consequence of the substitution of (31) in the general relation

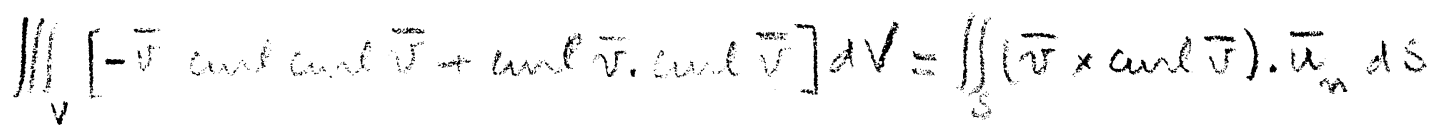

The right-hand member of (35) vanishes for all admissible vectors. As a consequence, $J(\bar{c})$ can be rewritten as $J(\bar{c})=\frac{N}{g}$

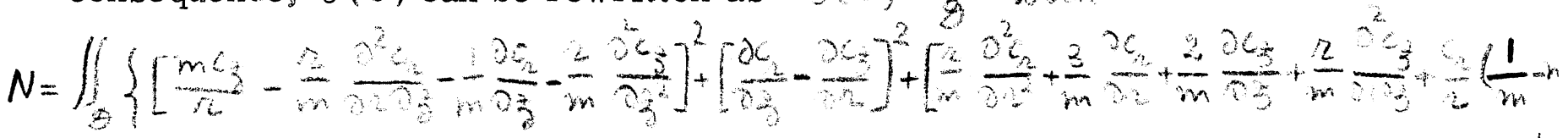

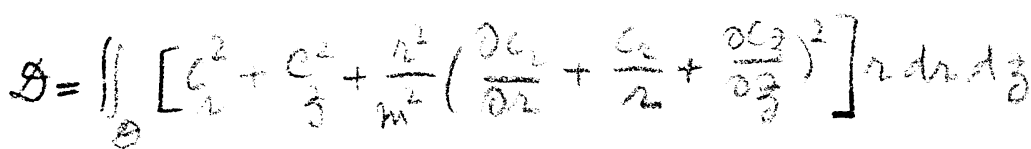

This form is suitable for numerical computations. We repeat that the admissible vectors must satisfy the conditions

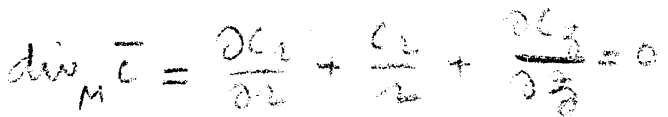

$$
\begin{aligned}
& \left|\bar{c} \times \bar{a}_{n}\right|=c_{2} \operatorname{Anz} z \cdot c_{0} \cos \theta 0
\end{aligned}
$$

at the boundary.

Finally, the normalization relations are

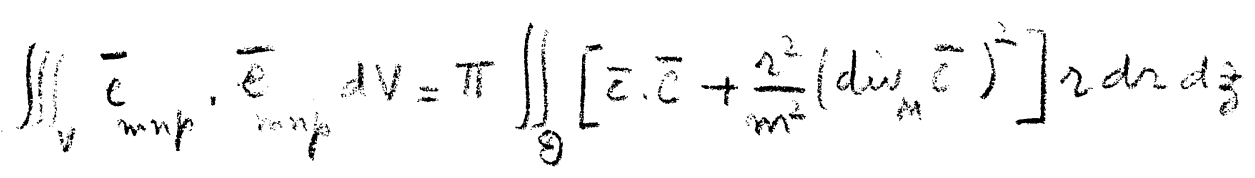

\section{EXPANSION IN ELECTRIC EIGENVECTORS}

We now turn to the task of determining the coefficients of expansion of the vector function $\bar{a}$ considered in the first paragraph. Written in full, the expansion has the form:

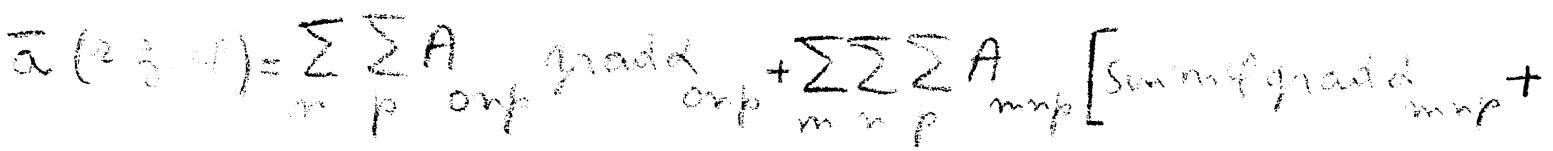

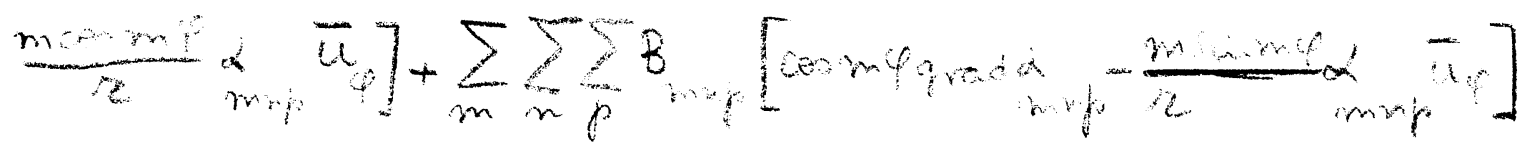


MURA-481

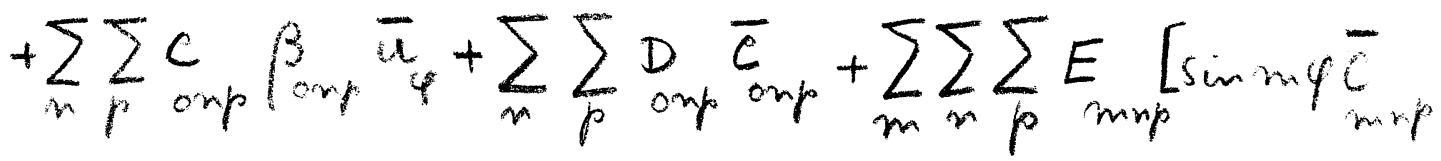

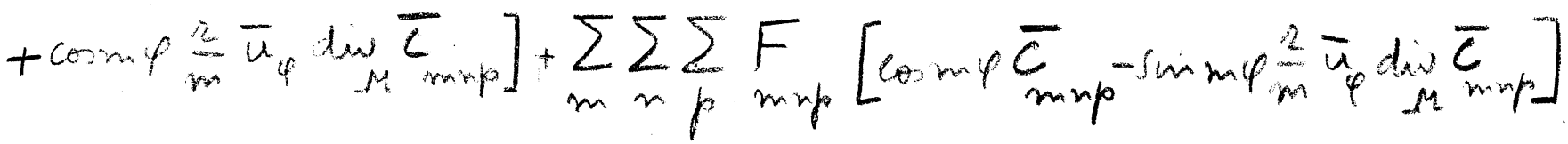

A comparision of this expansion with the Fourier series for $\bar{a}$, as given in (1), shows the meaning of certain groups of terms in (39).

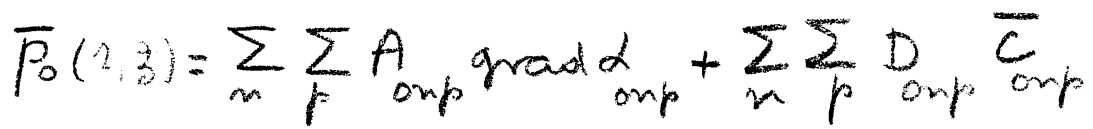

$$
\begin{aligned}
& v_{0}(2, z)=\sum_{n} \sum_{p} c_{\text {ort }} \beta_{\text {orr }}
\end{aligned}
$$

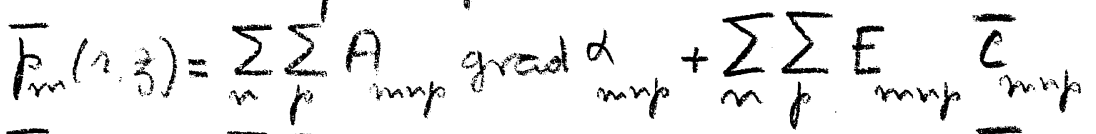

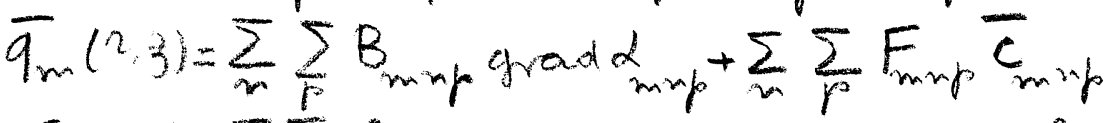

$$
\begin{aligned}
& v_{m}(2 j)=\sum_{r} \sum_{p} A_{m p p} \frac{m}{2} \alpha_{m i p}+\sum_{m} \sum_{p} E_{m p p} \frac{\Omega}{m} d \omega_{\mu} \bar{C}_{m p p}
\end{aligned}
$$

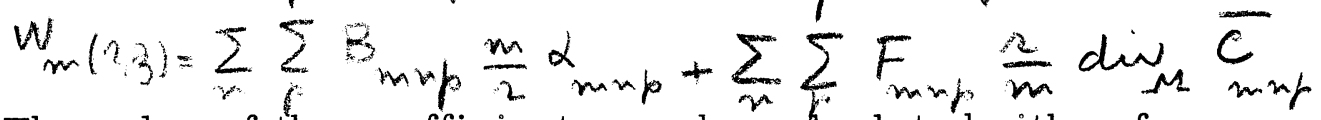

The value of the coefficients can be calculated either from general formulas, * or directly from (39) and (40). Results only will be quoted. For the irrotational terms:

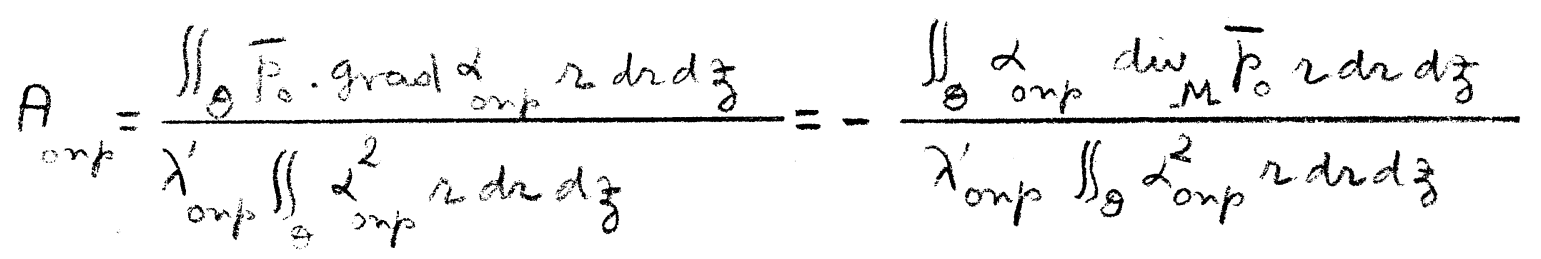

* The coefficient of $\bar{f}_{m x}$ is

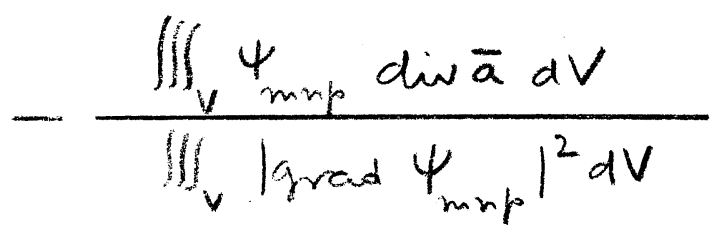

The coefficient of $\bar{l} \overline{m p p}_{\text {is }}$ is

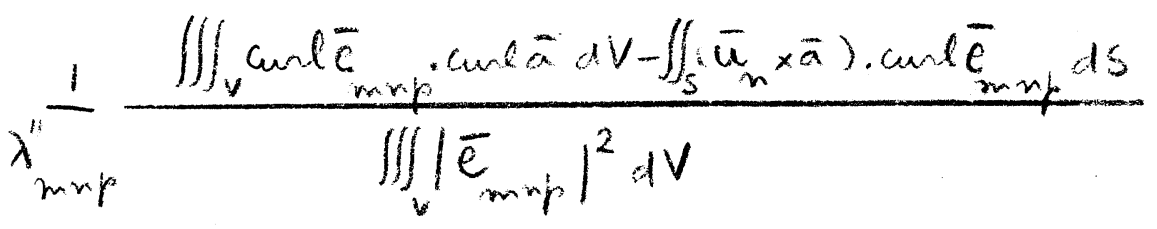

16 


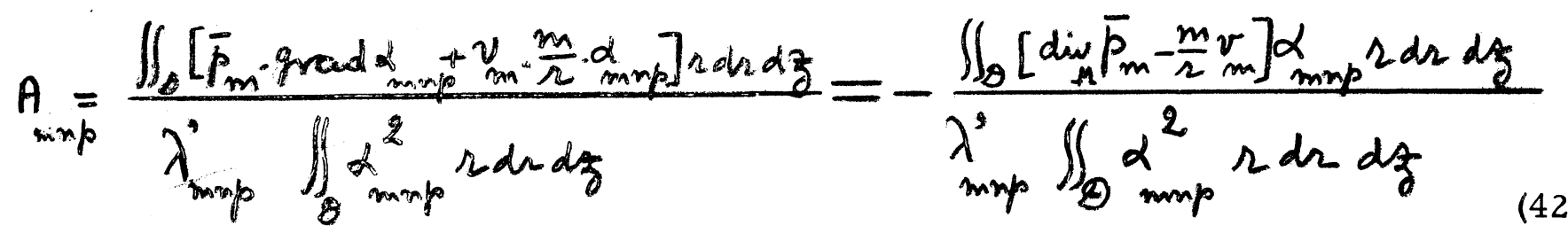

A similar expression can be obtained for $B_{m n p}$ by substituting $\bar{q}_{m}$ and $w_{m}$ for $\bar{P}_{m}$ and $v_{m}$ respectively. Formulas $(2),(41)$ and (42) indicate that coefficients $\mathrm{A}$ and $\mathrm{B}$ vanish when $\bar{a}$ is solenoidal. This result can be predicted directly from the general formulas.

For the solenoidal terms:

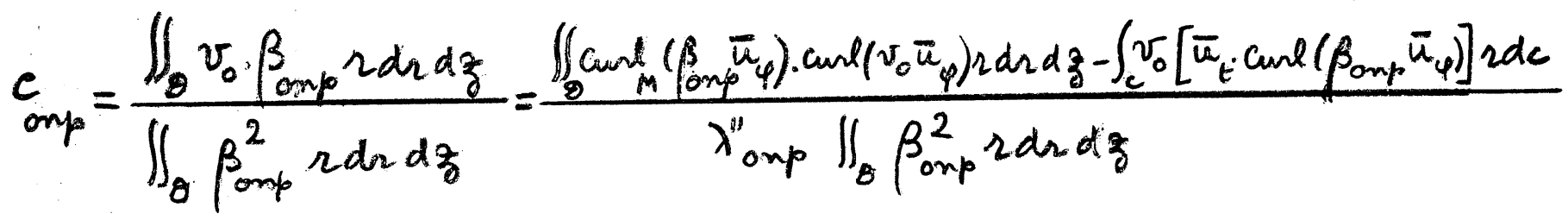

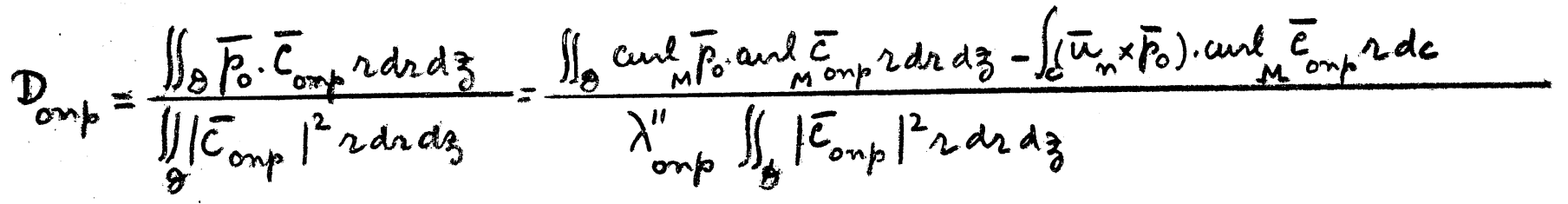

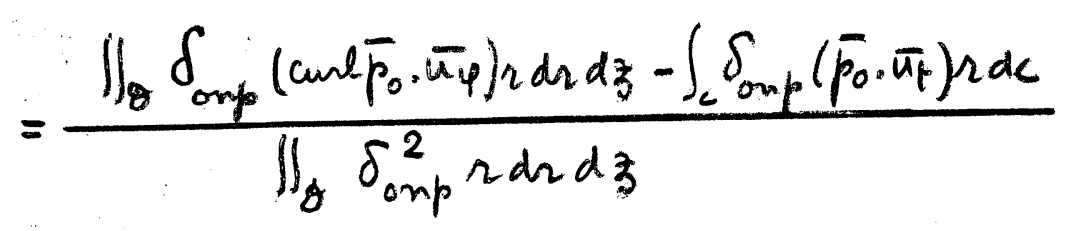

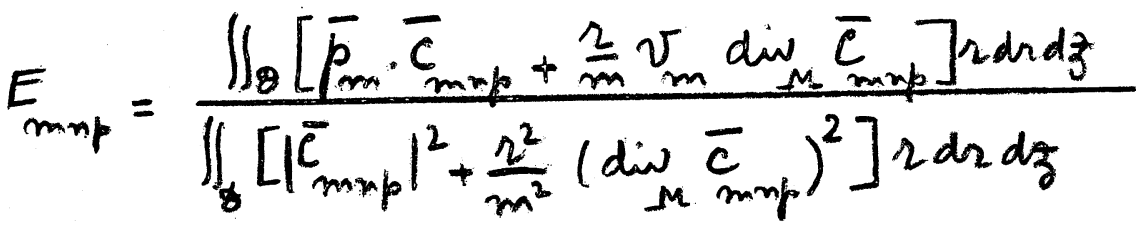

The numerator can be rewritten as

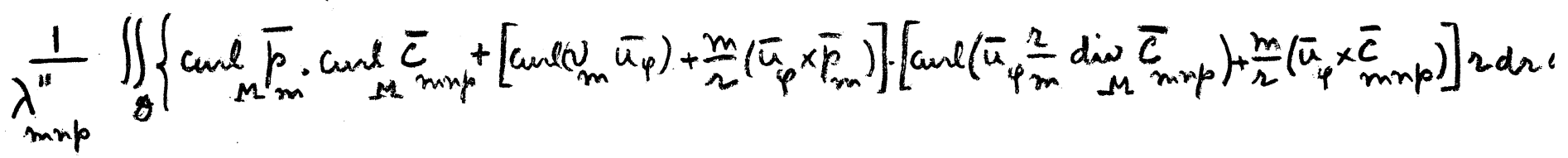

by an application of formulas (A1,12) and (A1,17) where $g$ is set equal to $\frac{\Omega}{m} \operatorname{div} \bar{c}_{m n p}$. 
A similar expression can be obtained for $F_{m \mathrm{~m} p}$ by substituting $\bar{q}_{\mathrm{m}}$ and $W_{m}$ for $\bar{p}_{m}$ and $v_{m}$ respectively. It will be noticed that the $C, D, E$ and $F$ vanish when $\bar{a}$ is irrotaticnal (which, according to formula (3), entails vanishing of the surface integral in the nume rator), and perpendicular to the boundary (which entails $\bar{\pi}_{m} \times \bar{p}_{m}=0$ and $v_{m}=0, i_{0} e_{0}$, cancels out the line integral in the numeratcr). These result,s are, again, in full agreement with the general formulas.

\section{MAGNETIC MODES IN TOROIDAL VOLUMES OF REVOLUTION}

The complete set of eigenvectors includes, first of all, a "sourceless" vector $\frac{\bar{u}_{\varphi}}{r}$. It includes, in addition, a triple infinity of irrotational eigenvectors and a triple infinity of solenoidal eigenvectors. These we now proceed to investigate.

\section{(a) Irrotational eigenvectors}

\section{a. 1. Modes of revolution}

The $\varphi$ independent modes $\bar{g}$ are gradients of the eigenfunctions of the problem

$$
\nabla_{M}^{2} \gamma_{\text {onp }}+\nu_{\text {onp }} \gamma_{\text {onp }}=0 \quad \frac{\partial \gamma_{\text {oup }}}{\partial n}=0 \text { on } C
$$

All the properties which were mentioned for the electric eigenfunctions (self-adjointress, orthogonality, norm) are still valid here。 (See II a.1) The eigenvalues can still be obtained as stationary value of (14), but the admissible functions are now required to have zero normal derivative on contour (c). It will be noticed that the lowest eigenfunction is actually $\gamma_{\text {onp }}=$ constant. This eigenfunction has zero gradient, and is of no interest to us. Higher eigenfunctions oniy must be considered. The trial functions for the latter must be 
orthogonal to $Y_{\text {oxp }}=$ constant, which implies satisfaction of the relation $\iint_{\partial} \gamma \Omega d r$

a. 2 Azimuth-dependent modes:

The $\varphi$ dependent modes are of the form $\bar{g}_{m \times p}=\operatorname{grad}\left[\sin m \varphi \gamma_{\text {mnp }}(2, z\right.$ where the are eigenfunctions of problem $(16)$, but with the boundary conditi $\frac{\partial \gamma_{m n p}}{\partial n}=0$ on c. All properties of the $\alpha$ mnp (orthogonality, norm, etc。) are still valid provided $\gamma$ and $\nu$ are substituted for $\alpha$ and $\lambda^{\prime}$ respectively. The eigenvalues can be obtained from $(18)$, but the admissible functions are now required to have zero normal derivative on (c).

(b) Solenoidal eigenvectors

b. 1 Modes of revolution

Two categories of modes will be recognized here。

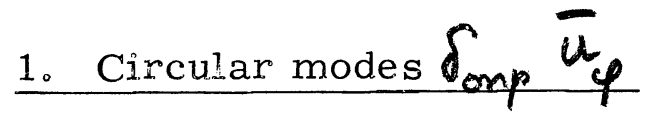

There is a double infinity of these modes, corresponding to the eigenfuncticns of problem (23) with accompanying boundary conditions. The normalization relation is simply

$$
\begin{aligned}
& \iiint_{V} \delta \bar{u}_{\varphi} \cdot \delta \bar{u}_{\varphi} d V=2 \pi \iint_{\theta_{-}} \delta^{2} r d r d z \\
& \text { 2. Meridian modes } d_{\text {onp }}
\end{aligned}
$$

These eigenvectors are actually the curl of the circular electric

eigenvectors. In mathematical form:

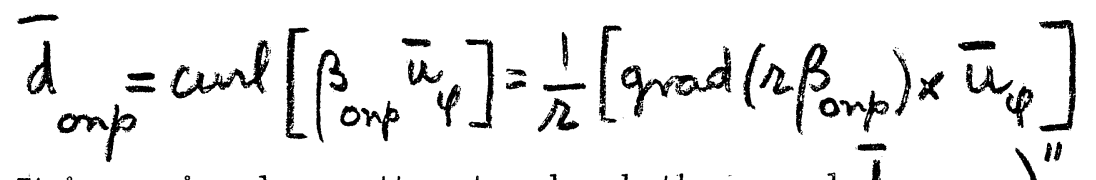

It is a simple matter to check that curl $\bar{d}_{\text {onp }}=\lambda_{\text {onp }}^{\prime \prime} \beta_{\text {omp }} \bar{u}_{\varphi}$ in consequence curl $\bar{d}$ vanishes on the surface of the torus, and the boundary 
condition $\bar{u}_{m} \times \operatorname{ant} \bar{d}=0$ is satisfied there, as it should.

The normalization integral is simply

$$
\begin{gathered}
\iiint_{V} \bar{d}_{o n p} \cdot \bar{d}_{o x p} d V=2 \pi \lambda_{o n p}^{\prime \prime} \iint_{\theta} \beta_{o x p}^{2} r d r d z \\
\text { b.2 Azimuth-dependent modes. }
\end{gathered}
$$

The magnetic vectors are the curl of the electric vectors $\bar{e}$ mmp . More precisely, with $\bar{e}_{\text {mup }}$ given by (31), $\bar{h}_{\text {mup }}$ will be

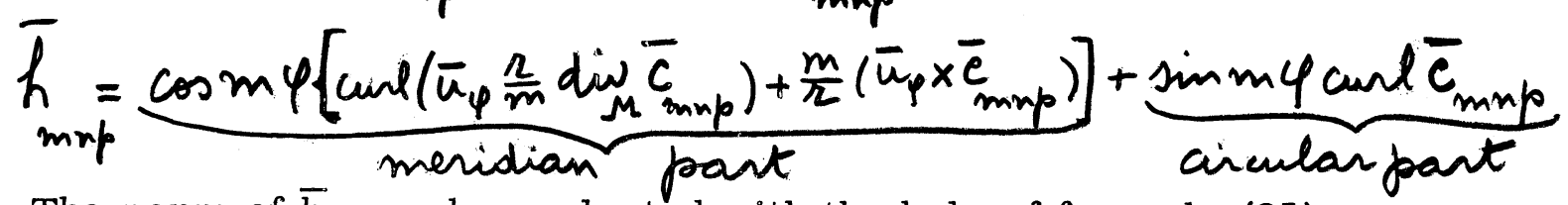

The norm of $\bar{h}$ can be evaluated with the help of formula (35), as:

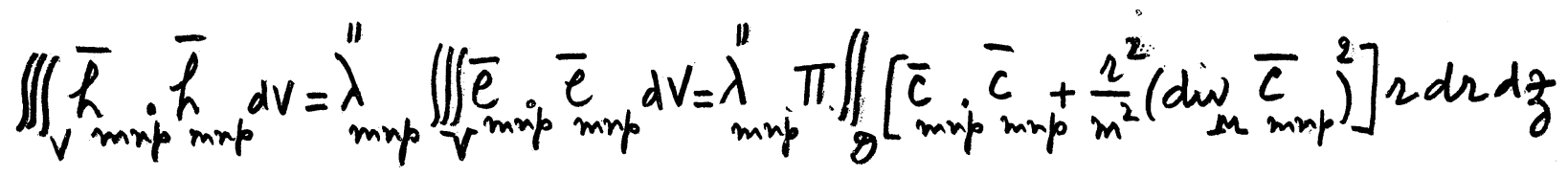

If $\bar{l}$ has been previously normalized, the normalized magnetic eigenvector is $\frac{\operatorname{cunl}_{\bar{m}} \overline{e_{m p}}}{\sqrt{\lambda_{\min }^{*}}}$. It is sometimes desirable to calculate $\frac{\bar{h}}{\operatorname{mnp}} \operatorname{directly}$ without relying on a previous knowledge of $\bar{e}_{m n p}$. The relevant steps are as follows:

1. $\bar{h}$

$$
\prod_{m n p}=\bar{d} \sin m \varphi+\frac{r}{m} \cos m \varphi \bar{u} \bar{\varphi}_{\varphi} \operatorname{div}_{\mu} \bar{d}
$$

2. If we go through the same motions as with the electric eigenvectors, we discover that the meridian part $\bar{d}_{\min }$ is an eigenvector of the problem

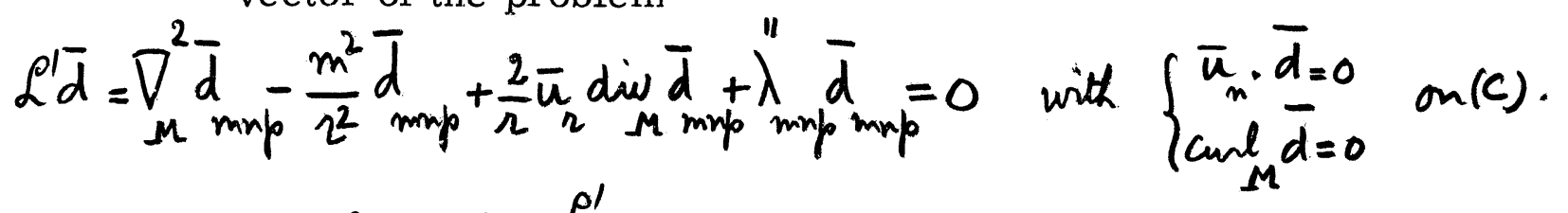
Transformation $\mathcal{L}^{\prime}$ is again self-adjoint and negative-definite with respect to scalar product (33). Its eigenvectors are orthogonal, 
ard its eigenvalues are the stationary values of (34) or

(36). The admissicle vectors, however, must now satisfy

the boundary conditions as evidenced in (51). These can

be written more explicitly as

$$
\begin{aligned}
& \bar{u}_{n} \cdot \bar{d}=d_{\Omega} \cos \varepsilon+d_{z} \sin \varepsilon=0 \\
& |\underset{\mu}{\text { curl } \bar{d}}|=\frac{\partial d_{\Omega}}{\partial z}-\frac{\partial d_{z}}{\partial \Omega}=0
\end{aligned}
$$

In the process of establishing (36) from (34), relation (35) must be used, and it is necessary to ascertain that the right-hand member of the latter relation vanishes for all admissible vectors. This boils down to a proof that

curl ${ }_{M}\left(\frac{2}{m} \bar{u}_{\varphi} \operatorname{liw}_{M} \bar{d}\right)$ is directed along the normal to (c) whenever boundary conditions (52) are satisfied. The proof is fairly straightforward. First of all, $\operatorname{curl}_{M}\left(\frac{2}{m} \bar{u}_{\varphi} \operatorname{diw}_{M} \bar{d}\right)=\frac{1}{\eta m} \underset{M}{\operatorname{grad}}\left(r^{2} \operatorname{diw}_{M} \bar{d}\right) \times \bar{u}_{\varphi} \quad$, as can easily be checked by writing the various differential operators in full. We now have to establish that $\operatorname{grad}_{M}\left(r^{2} d \dot{w}_{M} \bar{d}\right)=r^{2} \operatorname{grad}_{M} \operatorname{diw}_{M} \bar{d}+2 \pi \bar{u}_{\Omega} \operatorname{diw}_{M} \bar{d} \quad$ is tangent to (c). But this is an immediate consequence of $(51)$, because

$$
r^{2}\left(\operatorname{grad}_{M} \operatorname{div}_{M} \bar{d}+\frac{2}{2} \bar{u}_{\Omega} \operatorname{din}_{M} \bar{d}\right)=r^{2}\left(\frac{m^{2}}{1^{2}} \bar{d}-\lambda^{\prime \prime} \bar{d}+\operatorname{cunl}_{M} \text { cunl } \bar{d}\right)
$$

and the right-hand member is tangent to (c). More precisely:

(a) $\bar{d}$ is tangent to (c),

(b) curl ${ }_{M} \operatorname{curl}_{M} \bar{d}$ is tangent to (c). To prove this point, notice that $\operatorname{curl}_{M}^{M} \bar{d}=\left(\frac{\partial d_{r}}{\partial z}-\frac{\partial d_{\xi}}{\partial r}\right) \bar{u}_{\varphi}$. This vector is of the form $\beta \bar{u}_{\varphi}$. with $\beta=0$ along co But curl $\left(\beta \bar{u}_{\varphi}\right)=\frac{1}{\Omega}\left[\underset{\mu}{\operatorname{arad}}(\beta \Omega) \times \bar{u}_{\varphi}\right]$. The gradient of $\beta \Omega$ is perpendicular to $(c)$, and, as a consequence, $\operatorname{curl}_{M}\left(\beta \bar{u}_{\varphi}\right)=\mathrm{curl}_{M} \mathrm{cull}_{\mathcal{M}} \bar{d}$ is tangent to co 
MURA-481

V. EXPAINSION IN MAGNETIC EIGENVECTORS.

The expansion of a vector $\bar{a}$ has the form

$$
\begin{aligned}
& \bar{a}(r, z, \varphi)=A_{0}^{0} \frac{\bar{u}_{0 \varphi}}{n}+\sum_{n} \sum_{p} A_{\text {onp }}^{\prime} \text { grad } \gamma_{\text {onp }}+\sum_{m} \sum_{n} \sum_{p} A_{\text {mnp }}^{\prime}\left[\sin m \varphi \text { grad } \gamma_{\text {minp }}\right. \\
& \left.+\frac{m \cos m \varphi}{n} \gamma_{m \times p} \bar{u}_{\varphi}\right]+\sum_{m} \sum_{n} \sum_{p} B_{m i p}^{\prime}\left[\cos m \varphi g r a d \gamma_{m x p}-\frac{m \sin m \varphi}{n} \gamma_{\min } \bar{u}_{\varphi}\right] \\
& +\sum_{n} \sum_{p} c_{\text {onp }}^{\prime} \delta_{\text {onp }} \bar{u}_{\varphi}+\sum_{n} \sum_{p} D_{\text {onp }}^{\prime} \bar{d}_{\text {onp }} \\
& +\sum_{m} \sum_{n} \sum_{p} E_{\operatorname{mpp}}^{\prime}\left[\sin m \varphi \bar{d}_{m x p}+\operatorname{cosm} \varphi \frac{r}{m} \bar{u}_{\varphi} d \omega_{\mu} \bar{d}_{m p r}\right] \\
& +\sum_{m} \sum_{n} \sum_{p} F_{m n p}^{\prime}\left[\cos m \varphi \bar{d}_{m p p}-\sin m \varphi \frac{r}{m} \bar{u}_{\varphi} \operatorname{di⿻}_{\mu} \bar{d}_{m p p}\right]
\end{aligned}
$$

A comparison with the Fourier expansion for $\bar{a}_{2}$ as given in (1), leads to the relations

$$
\begin{aligned}
& \bar{p}_{0}(r, \xi)=\sum_{n} \sum_{p} A_{\text {oup }}^{\prime} \text { grad } \gamma_{\text {oup }}+\sum_{m} \sum_{p} D_{\text {onp }}^{\prime} \bar{d}_{\text {orp }} \\
& v_{0}(r, \xi)=\frac{A_{0}^{\prime}}{r}+\sum_{n} \sum_{p} e_{\text {oup }}^{\prime} \delta_{\text {orp }}
\end{aligned}
$$

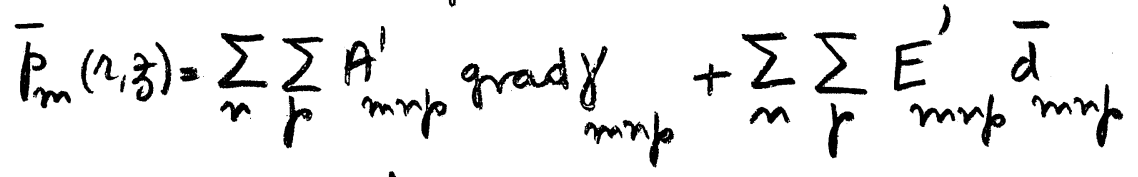

$$
\begin{aligned}
& \bar{q}_{m}(1, \xi)=\sum_{n} \sum_{p} B_{\text {mup }} \dot{g r a d}_{\text {mup }}+\sum_{n} \sum_{p} F_{\text {mnp }}^{\prime} \bar{d}_{\text {mnp }} \\
& v_{m}(n, \xi)=\sum_{n} \sum_{p} A_{m r p}^{\prime} \frac{m}{n} \gamma_{m m p}+\sum_{m} \sum_{p} E_{m m p}^{\prime} \frac{n}{m} d w_{\mu} \bar{d}_{m p p} \\
& w_{m}(2, \xi)=\sum_{m} \sum_{p} B_{m p p}^{\prime} \frac{m}{\pi} \gamma_{m p p}+\sum_{m} \sum_{p} F_{m m p}^{\prime} \frac{n}{m} \operatorname{din}_{m} \bar{d}_{m p r}
\end{aligned}
$$

22 
The value of the coefficients can be calculated either from general formulas ${ }^{*}$, or directly from (53) and (54). Results are, for the sourceless vector,

$$
A_{0}^{\prime}=\frac{\iint_{\theta} v_{0} d r d z}{\iint_{\theta} \frac{d r d z}{2}}
$$

$v_{0}(r, z)$ is $\frac{1}{2 \pi r}$ times the circulation of $\bar{a}$ around the "parallel" circle through $r, \xi$. For a vector which is irrotational in the toroidal region, the circulation is constant and equal to $2 \pi \mathrm{A}_{0}^{\prime}$.

$$
\begin{aligned}
& \text { For the irrotational vectors, the coefficients are } \\
& A_{\text {onp }}^{\prime}=\frac{\int_{c} \gamma_{\text {onp }}\left(\bar{u}_{n} \cdot \bar{p}_{0}\right) r d c-\iint_{\theta} \gamma_{\text {onp }} d \operatorname{liv}_{m} \bar{p}_{0} r d r d \bar{z}}{\nu_{\text {onp }} \iint_{\theta} \gamma_{\text {onp }}^{2} r d r d z} \\
& A_{m i p}^{\prime}=\frac{\int_{c} \gamma_{m m p}\left(\bar{u}_{m} \cdot \bar{p}_{m}\right) r d c-\iint_{\theta} \gamma_{m m p}\left(\operatorname{din}_{m} \bar{p}_{m}-\frac{m}{r} v_{m}\right) r d r d z}{\nu_{m n p} \iint_{B} \gamma_{m p}^{2} r d r d z}
\end{aligned}
$$

A similar expression can be obtained for $B_{m n p}^{\prime}$ by substituting $\bar{q}_{m}$ and $W_{m}$ for $\bar{P}_{m}$ and $v_{m}$ respectively. Formulas (2) and (56) indicate that $A^{\prime} A_{\text {on }}^{\prime}$ and $B^{\prime}$ vamish wher $\bar{a}$ is sclenoidal and perpendicular to the boundary. This result can also be deduced directly from the general formulas.

For the sclenoidal vectors, using formula A1.17

$c_{\text {onp }}^{\prime}=\frac{\iint_{0} v_{0} \delta_{\text {onp }} r d r d z}{\iint_{0} \delta_{\text {onp }}^{2} r d r d z}=\frac{\iint_{\theta} \operatorname{aul} l\left(\delta_{\text {onp }} \bar{u}_{\varphi}\right) \cdot \operatorname{curl}\left(v_{0} \bar{u}_{\varphi}\right) r d r d z}{\lambda_{\text {onp }}^{\prime \prime} \iint_{z} \delta_{\text {onp }}^{2} r d r d z}$

* The coefficient of $\bar{h}_{0}$ is $\quad \frac{\iint_{V} \bar{a} \cdot \bar{h}_{0} d V}{\iiint_{V} \bar{h}_{c} \cdot \bar{h}_{0} d V}$

The coefficient of $\bar{g}_{\text {mnp }}$ is $-\frac{\iint_{S}\left(\bar{u}_{n} \cdot \bar{a}\right) \theta_{\text {mup }} d S-\iiint_{V} \theta_{\text {mip }} d i \bar{a} d V}{\iiint_{V} \mid \text { qad }\left.\theta_{\operatorname{mnp}}\right|^{2} d V}$

The coefficient of $\bar{h}_{m n p}$ is

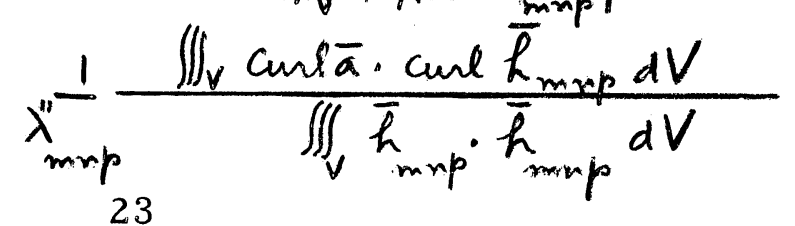




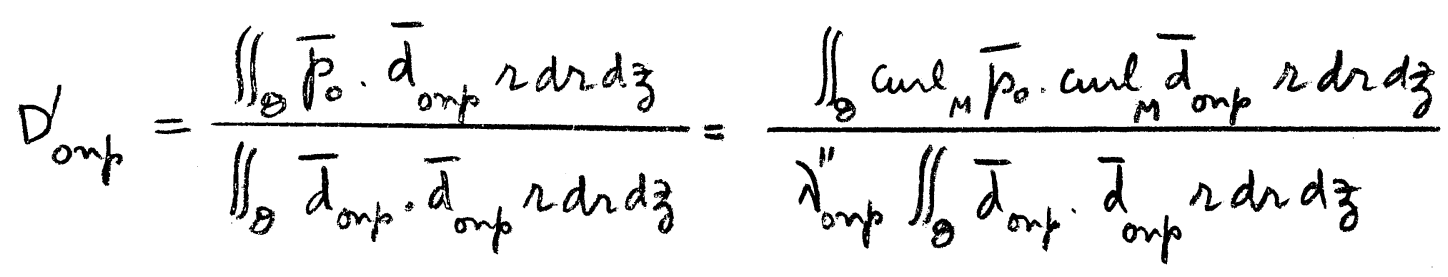

$$
\begin{aligned}
& =\frac{\iint_{\theta} \beta_{\text {oup }}\left(\operatorname{aull} \bar{p}_{0} \cdot \bar{u}_{\varphi}\right) r d r d z}{\iint_{\theta} \beta_{o u p}^{2} r d r d z}
\end{aligned}
$$

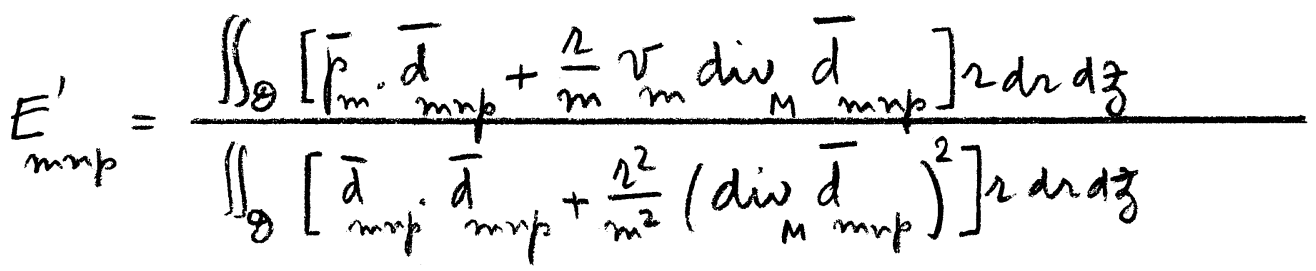

The numerator can be rewritten as

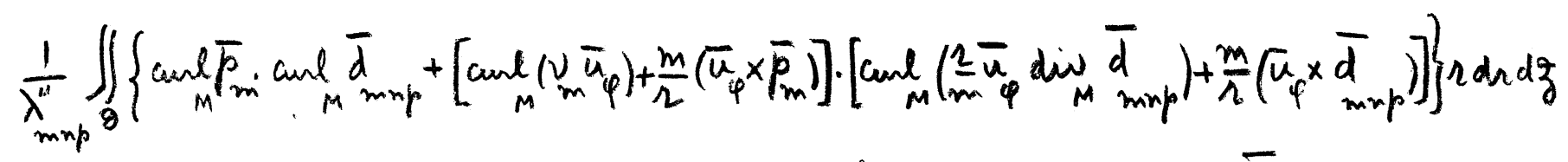

A similar expression can be obtained for $F^{b}{ }_{m n p}$ by substituting $\bar{q}_{m}$ and $W_{m}$ for $\bar{P}_{m}$ and $v_{m}$ respectively. It will be noticed that, according to formula (3), the $C^{\natural} D^{\imath} E^{\imath}$ and $F^{\natural}$ vanish when $\bar{a}$ is irrotational. This result is, again, in harmony with the predictions of the general formulas.

\section{REGIONS CONTAINING THE AXIS}

In regions of the type depicted in Fig. $1 \mathrm{~b}$ and $1 \mathrm{c}$, which contain parts of the axis of revolution, the Fourier expansion coefficients of a continuous function $A(r, g, \varphi)$ behave in an interesting way in the vicinity of the axis. 
Let the expansion be written as

$$
A(r, z, \varphi)=A_{0}(n, z)+\sum_{m=1}^{\infty} \sin m \varphi \underset{m}{A}(r, z)+\sum_{m=1}^{\infty} \cos m \varphi B_{m}(n, z)
$$

If $A$ is continuous at all points, including those situated on the axis, the limit of A as $r$ approaches zero must be independent of $\varphi$. This clearly requires $A_{m}$ and $\mathrm{B}_{\mathrm{m}}$ to vanish on the axis, while the value of $A$ reduces to $\mathrm{A}_{0}(0, \xi)$ thereon.

Consider now a vector $\overline{\mathrm{a}}$, continuous at all points, including those situated on the axis, and possessing a Fourier expansion of the type given in equation (1). By a series of simpie calculations, the details of which are presented in Appendix III, it is possible to establish the following properties of the Fourier coefficients:

(a) po is directed along the axis

(b) $v_{0}$ vanishes on the axis

(c) $\bar{p}_{1}$ and $\bar{q}_{1}$ are purely radial on the axis, and the equalities

$$
p_{1 r}=v_{1}, q_{1 r}=w_{1} \text { hold there. }
$$

(d) The coefficients $\bar{p}_{m}, \bar{q}_{m}, v_{m}$ and $W_{m}$ vanish on the axis when $m$ is larger than one.

These simple rules for scalar and vector functions allow one to foresee the behavior of functions possessing higher orders of continuity. For example:

1. If the gradient of $A$ is continuous on the axis,

$$
\begin{aligned}
& \text { (a) } \frac{\partial A_{0}}{\partial r}=0 \\
& \text { (b) } A_{1}=B_{1}=\frac{\partial A_{1}}{\partial z}=\frac{\partial B_{1}}{\partial z}=0 \\
& \text { (c) } A_{m}=B_{m}=\frac{\partial A_{m}}{\partial z}=\frac{\partial B_{m}}{\partial r}=\frac{\partial A_{m}}{\partial z}=\frac{\partial B_{m}}{\partial \xi}=0 \quad(m>1) \\
& \text { on the axis. }
\end{aligned}
$$


2. If the divergence of $\bar{a}$ is continuous on the axis, the properties
(a) $\frac{\partial v_{1}}{\partial r}=\frac{\partial w_{1}}{\partial r}=0$
(b) $\frac{\partial p_{m}}{\partial r}=\frac{m}{2} \cdot \frac{\partial v_{m}}{\partial r}$ and $\frac{\partial q_{m r}}{\partial r}=\frac{m}{2} \cdot \frac{\partial w_{m}}{\partial r}$

hold on the axis (in addition to those mentioned above).

3. If the curl of $\bar{a}$ is continuous on the axis, the properties
(a) $\frac{\partial p o z}{\partial r}=0$
(b) $\frac{\partial p_{1 n}}{\partial r}=2 \frac{\partial v_{1}}{\partial r}$ and $\frac{\partial q_{i n}}{\partial r}=2 \frac{\partial w_{1}}{\partial r}$
(c) $\frac{\partial p_{m \xi}}{\partial r}=\frac{\partial q_{m \xi}}{\partial r}=0$ and $\frac{\partial U_{m}}{\partial r}=\frac{m}{2} \cdot \frac{\partial p_{m r}}{\partial r}, \frac{\partial w_{m}}{\partial r}=\frac{m}{2} \cdot \frac{\partial q_{m r}}{\partial r}$

hold on the axis in addition to those mentioned above.

In the solution of eigenfunction equations such as (6) and (7), it is necessary, for practical computational purposes, to have as much information about the behavior of the functions on the axis as possible. Scalar and vector eigenelements must have continuous Laplacian and curl curl respectively. What this implies can easily be found out by combining results obtained above. One finds out that:

1. For a scalar function with continuous Laplacian
(a) $\frac{\partial A_{0}}{\partial L}=0$
(b) $A_{1}=B_{1}=\frac{\partial^{2} A_{1}}{\partial r^{2}}=\frac{\partial^{2} B_{1}}{\partial r^{2}}=0$
(c) $A_{m}=B_{m}=\frac{\partial A_{m}}{\partial r}=\frac{\partial B_{m}}{\partial r}=\frac{\partial^{2} A_{m}}{\partial r^{2}}\left(\frac{m^{2}}{4}-1\right)=\frac{\partial^{2} B_{m}}{\partial r^{2}} \cdot\left(\frac{m^{2}}{4}-1\right)$

2. For a vector function with continuous curl curl
(a) $p_{o r}=\frac{\partial p_{0 z}}{\partial r}=v_{0}=\frac{\partial^{2} v_{0}}{\partial n^{2}}=0$
(b) $p_{1}=0 \quad \frac{\partial p_{12}}{\partial q_{2}}=2 \frac{\partial w_{1}}{\partial n^{\prime}}$ (and similar relations for $\overline{q_{1}}$ and $w_{1}$ ) 


$$
\text { (c) } p_{m}=p_{m z}=\frac{\partial p_{m g}}{\partial r}=V_{m}=0 \text { and } \frac{\partial U_{m}}{\partial r}=\frac{m}{2} \cdot \frac{\partial p_{m}}{\partial r}
$$

on the axis. Similar relations can be written for $\bar{q}$ and $w_{m}$.

We are now in a position to more accurately set up the eigenfunction problems for a simply-bounded, simply-connected cavity of the type shown in Figure $1 \mathrm{~b}$.

For both electric and magnetic irrotational eigenvectors:

(a) For modes of revolution, as described in equations (12) and (44), the boundary conditions on the outer contour are unchanged, but one should add the condition

$$
\frac{\partial \alpha}{\partial r}=\frac{\partial \gamma}{\partial r}=0 \quad \text { on the axis. }
$$

(b) For azimuth-dependent modes, as described in equation (16) one should add the condition $\alpha=\gamma=0$ on the axis.

For both electric and magnetic solenoidal eigenvectors:

(a) For circular modes of revolution, as described in equations (19) and (23), one should add the condition

$$
\beta=\delta=0 \quad \text { on the axis. }
$$

(b) For meridian modes of revolution, as described in (21) and (46), one should add the condition

$$
c_{r}=\frac{\partial C_{3}}{\partial r}=0 \quad \text { on the axis. }
$$

(c) For azimuth dependent modes, as described in (30) and (51), one should add the conditions

$$
\begin{array}{ll}
c_{z}=\frac{\partial C_{r}}{\partial r}=0 & \text { for } m=1 \\
c_{z}=c_{2}=0 & \text { for } m>1 \quad \text { on the axis. }
\end{array}
$$

These various relations will be checked on the normal modes of the circular cylinder, which can be written down explicitly by separation of variables. 
Before this is done, however, it is necessary to mention that, in a doubly bounded volume such as represented in Figure 1c, the electric eigenvectors mentioned above do not form a complete set unless we add the electrostatic field grad $\alpha_{0}$ to them. This field is obtained by establishing a potential difference between the two boundary surfaces, assumed to be metallized. More precisely, $\alpha_{0}$ is the solution of

$$
\begin{aligned}
& \nabla_{M}^{2} \alpha_{0}=0 \\
& \alpha_{0}=1 \text { on } s_{1} \quad \alpha_{0}=0 \text { on } s_{2}
\end{aligned}
$$

(or any multiple thereof).

\section{APPLICATION TO THE CIRCULAR CYLINDRICAL CAVITY}

The eigenvectors for this cavity are well known. ${ }^{4}$ We first consider the electric eigenvectors. The irrotational set derives from scalar functions:
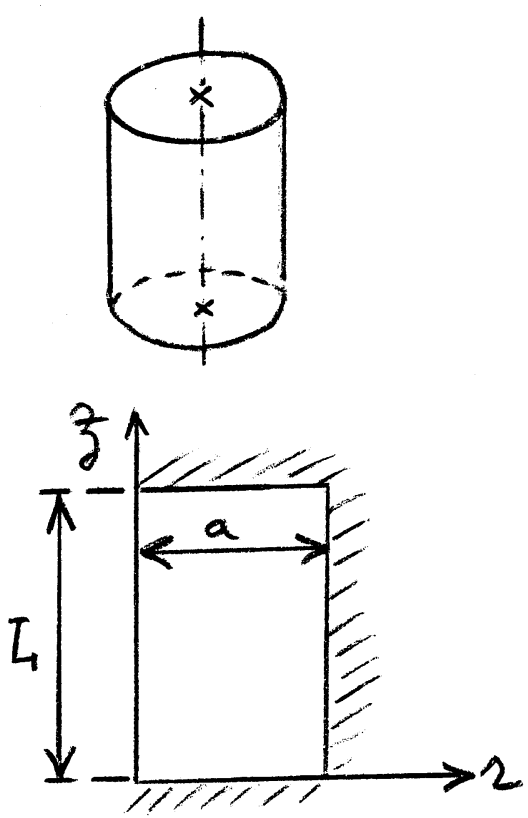

Fig. 2 (a) $\alpha_{\text {onp }}=\sin \frac{n \pi \xi}{L} \cdot J_{0}\left(\mu_{\text {op }} \frac{r}{a}\right)$

for modes of revolution. Numbers $\mu_{m p}$ are the roots of

$$
\operatorname{Jm}(x)=0
$$

The power expansion of Bessel's functions

$$
J_{m}\left(\lambda_{2}\right)=\frac{\left(\lambda_{2}\right)^{m}}{2^{m} m !}\left[1-\frac{(\lambda r)^{2}}{4(m+1)}+\cdots\right]
$$

shows clearly that the general condition $59 \mathrm{a}$, i.e. $\frac{\partial \alpha_{\text {oup }}}{\partial \Omega}=0$, is satisfied on the axis.

(b) $\alpha_{m n p}=\sin \frac{n \pi \xi}{L} J_{m}\left(\mu_{m p} \frac{\Omega}{a}\right)$

for azimuth-dependent modes. Clearly $\alpha_{\text {manp }}$ vanishes on the axis, while 4see e.g. C. G. Montgomery "Technique of Microwave Measurements" p. 297, McGraw Hill Book Company, New York, 1947. 
$\frac{\partial \alpha_{m m}}{\partial n}=0$ for $m>1$. The general conditions $59 \mathrm{~b}$ ) and c) are confirmed.

The solenoidal set is somewhat more complicated. We first consider modes of revolution. They are of two sorts:

(a) Circular modes:

$$
\beta_{\text {onp }} \bar{u}_{\varphi}=\sin \frac{n \pi \xi}{L} J_{1}\left(\mu_{1 p} \frac{n}{a}\right) \bar{u}_{\varphi}
$$

It will be noticed that $\mu_{1 p}$ is a root of $J_{1}(x)=0$, but also of $J^{\prime}{ }_{0}(x)=0$ The expansion of $J_{1}$ shows that condition 60 a) (i. e. $v_{0}=\frac{\partial^{2} v_{0}}{\partial r^{2}}=0$ ) is verified.

(b) Meridian modes:

$$
\bar{C}_{\text {orp }}=\operatorname{cunl}\left[\delta_{\text {onp }} \bar{u}_{\varphi}\right]=\operatorname{curl}\left[\cos \frac{n \pi \xi}{L} J_{1}(\lambda r) \bar{u}_{\varphi}\right]
$$

The boundary condition at $r=a$ is

$$
\frac{1}{a} \frac{\partial}{\partial r}\left[r J_{1}(\lambda r)\right]_{n=a}=\lambda J_{0}(\lambda a)=0
$$

indicating clearly that $\lambda$ is equal to $\frac{\mu_{\text {op }}}{a}$. Written in full, the eigenvector is $\bar{C}_{\text {onp }}=\frac{n \pi}{L} \cdot \sin \frac{n \pi z}{L} J_{1}\left(\mu_{o p} \frac{n}{a}\right) \bar{u}_{n}+\frac{\mu_{o p}}{a} \cos \frac{n \pi z}{L} J_{0}\left(\mu_{o p} \frac{r}{a}\right) \bar{u}_{z}$

Conditions $p_{o r}=\frac{\partial p_{0 z}}{\partial r}=0$, as given in (60a), are satisfied. For azimuthdependent modes, one obtains the formula

$\bar{c}=-\frac{n \pi}{L} \cdot \frac{a^{2}}{\mu_{m p}^{2}} \cdot \sin \frac{n \pi \xi}{L} \cdot \frac{d J_{m}\left(\mu_{m p} \frac{\Omega}{a}\right)}{d r} \bar{u}_{n}+\cos \frac{n \pi \xi}{L} J_{m}\left(\mu_{m p} \frac{\Omega}{a}\right) \bar{u}_{z}$

The $(r, z)$ dependent part of the circular component is

$$
v_{m}(r, z)=\frac{r}{m} \operatorname{diw}_{M} \bar{C}_{\operatorname{manp}}=-\frac{n \pi}{L} \cdot \frac{m}{r} \cdot \frac{a^{2}}{\mu_{m p}^{2}} \cdot \sin \frac{n \pi z}{t} \cdot J_{m}\left(\mu_{m p} \frac{r}{a}\right)
$$

The conditions $c_{z}=p_{m \xi}=0$ are satisfied for all values of $m$. In addition:

$$
\begin{aligned}
& \frac{\partial c_{n}}{\partial n}=\frac{\partial v}{\partial \Omega}=0 \text { for } \mathrm{m}=1 \\
& c_{r}=v=\frac{\partial c_{3}}{\partial n}=\frac{\partial v}{\partial \Omega}=\frac{\partial c_{n}}{\partial \Omega}=0 \text { for } \mathrm{m}>1
\end{aligned}
$$

by using formula (63) repeatedly. Conditions (60c) are satisfied. One could go 
through the same motions to check the form of the magnetic eigenvectors. We shall give a simple example only. The azimuth-dependent irrotational eigenvectors derive from scalar functions

$X_{\text {minp }}=\cos \frac{n \pi z}{L} J_{m}\left(b_{m p} \frac{a}{a}\right)$
where the numbers $b_{m p}$ are the roots of
$J_{m}^{\prime}(x)=0$

$$
J_{m}^{\prime}(x)=0
$$

Conditions (59) are satisfied. 


\section{Appendix I: Differential Operators in a Meridian Plane}

In the following equations $g, h, \bar{P}, \bar{P}, \bar{Q}$ are functions of $r$ and $z$ only, and $\bar{P}$ and $\bar{Q}$ are purely meridian vectors

1. $\operatorname{grad}_{\mu} g=\frac{\partial g}{\partial r} \bar{u}_{\nu}+\frac{\partial g}{\partial z} \bar{u}_{z}$

2. $\operatorname{dim}_{M} \bar{p}=\frac{\partial p_{r}}{\partial r}+\frac{p_{R}}{r}+\frac{\partial p_{z}}{\partial z}$

3. cunt $\bar{p} \bar{p}=-\frac{\partial p_{1}}{\partial z} \bar{u}_{n}+\left(\frac{\partial p_{r}}{\partial z}-\frac{\partial p_{z}}{\partial r}\right) \bar{u}_{\varphi p}+\left(\frac{p_{4}}{r}+\frac{\partial p_{4}}{\partial r}\right) \bar{u}_{z}$

4. $C_{\text {int }} \bar{p}=\left(\frac{\partial p_{2}}{\partial \gamma_{2}}-\frac{\partial p_{3}}{\partial r}\right) \bar{u}_{\varphi}$

$5 \cdot \nabla_{M}^{2} g=\frac{\partial^{2} g}{\partial r^{2}}+\frac{1}{\Omega} \cdot \frac{\partial g}{\partial r}+\frac{\partial^{2} g}{\partial z^{2}}=d_{M} \operatorname{qrad}_{M} g$

6 curl grad $g=0$

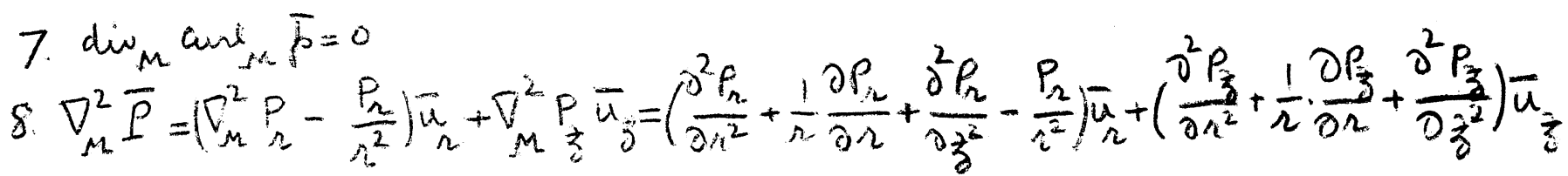

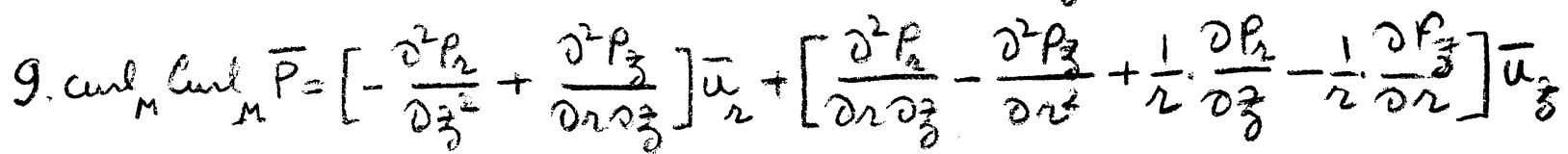

$$
=\underset{\mu}{\operatorname{grad}} \operatorname{diw}_{\mu} \bar{P}-\nabla_{\mu}^{2} \bar{P}
$$

10. $\nabla_{M}^{2} g=r \nabla_{M}^{2}\left(\frac{g}{r}\right)+\frac{2}{r} \cdot \frac{\partial g}{\partial r}+\frac{g}{r^{2}}=\frac{1}{r} \nabla_{\mu}^{2}(r g)-\frac{2}{r} \cdot \frac{\partial g}{\partial r}-\frac{g}{r^{2}}$

11. $\operatorname{div}_{M}\left(\nabla_{M}^{2} \bar{p}\right)=\nabla_{M}^{2}\left(\operatorname{div}_{M} \bar{P}\right)$

12. $\operatorname{div}_{M}\left[\nabla_{M}^{2} \bar{c}-\frac{m^{2} \bar{c}}{n^{2}}+\frac{2}{r} \bar{u}_{n} \operatorname{diw}_{M} \bar{c}\right]=\frac{m}{r} \nabla_{M}^{2}\left(\frac{r \operatorname{din}_{M} \bar{c}}{m}\right)+\frac{2 m^{2}}{n^{3}} c_{M}-\frac{m^{2}+1}{n^{2}} \operatorname{din}_{M} \bar{c}$

$$
=\nabla_{M}^{2}\left(\operatorname{div}_{M} \bar{c}\right)-\frac{m^{2}}{r^{2}} \operatorname{div}_{M} \bar{c}+\frac{2 m^{2}}{n^{3}} c_{r}+\frac{2}{2} \frac{\partial}{\partial r}\left(\operatorname{diw}_{M} \bar{c}\right)
$$

13. $\nabla_{M}^{2} \operatorname{grad}_{M} g=\operatorname{grad}_{\mu}\left(\nabla_{\mu}^{2} g\right)$ 
The followirg integral relations are directly obtainable from their threedimensional counterpart

14. $\iint_{\theta} \operatorname{diw}_{M} \bar{P} n d r d z=\int\left(\bar{u}_{n} \cdot \bar{P}\right)_{\Lambda} d c$

15. $\iint_{Q}\left[g \operatorname{din}_{M} \bar{p}+\right.$ grad $\left.g \cdot \bar{p}\right] r d r d z=\int_{C} g\left(\bar{u}_{n} \cdot \bar{p}\right)_{r} d c$

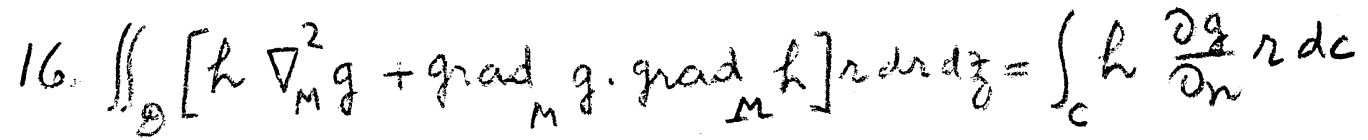

$17 \int_{\theta}\left\{\bar{Q}\left(\nabla_{M}^{2} \bar{P}-\frac{m^{2}}{r^{2}} \bar{P}+\frac{2 m g}{r^{2}} \bar{u}_{r_{0}}\right)+h\left(\nabla_{M}^{2} g-\frac{m^{2}+1}{r^{2}} g+\frac{2 m c_{r}}{r^{2}}\right)\right.$

$+\left(\operatorname{div}_{M} \bar{P}-\frac{m g}{\Omega}\right) \cdot\left(\operatorname{din}_{M} \bar{Q}-\frac{m h}{R}\right)+\operatorname{conl} \bar{P}_{M} \cdot \operatorname{coval}_{M} \bar{Q}$

$\left.+\left[\operatorname{avn}_{N}\left(\bar{u}_{\varphi}\right)+\frac{m}{r}\left(\bar{u}_{\varphi} \times \bar{p}\right)\right] \cdot\left[\operatorname{conl}\left(k \bar{u}_{\varphi}\right)+\frac{m}{\Lambda}\left(\bar{u}_{\varphi} \times \bar{Q}\right)\right]\right\} 2 d r d z$

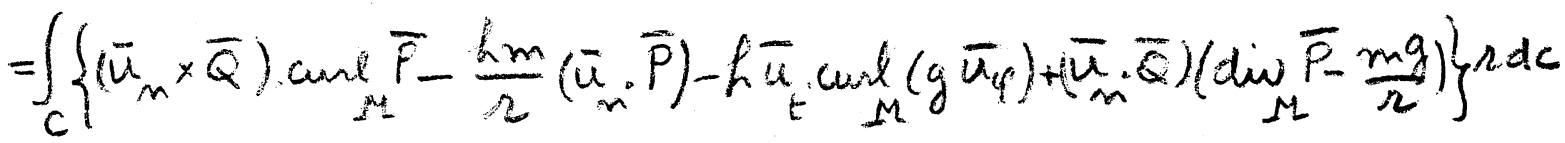




\section{APPENDIX II。 Properties of the operator $\mathcal{L}=\nabla_{\mu}^{2}-\frac{m^{2}}{r^{2}}+\frac{2}{r} \bar{u} \operatorname{div}_{\mu}$}

Scalar product (33), and the metric derived from it, define a Hilbert space. The mair properties of transformation $\mathcal{L}$ are obtained from a consideration of $\langle\bar{v}, \alpha \vec{v}\rangle$, where $\bar{v}$ belongs to the domain of vectors satisfying the boundary conditions appearing in (32). If we apply (35) to $\bar{v}$, we discover that the righthand member vanishes, so that

$$
\begin{aligned}
& \langle\bar{v}, f \bar{v}\rangle=\iint_{\theta}\left[\bar{v} \cdot L \bar{v}+\frac{r^{2}}{m^{2}} \operatorname{din}_{M} \bar{v} \cdot \operatorname{din}_{M} P \bar{v}\right] r d u d \bar{z} \\
& =-\| \int_{\partial}\left\{\left|\operatorname{mun}_{M} \bar{v}\right|^{2}+\left|\frac{m}{r} \bar{u}_{\psi} \times \bar{v}+\operatorname{cul}_{M}\left(\frac{r}{m} \bar{u}_{\varphi} d w_{M} \bar{c}\right)\right|^{2}\right\} r d r d z
\end{aligned}
$$

Clearly $\langle\bar{v}, L \bar{v}\rangle$ is never positive. We now want to prove that $\alpha \bar{v}=0$ implies $\bar{V}_{0}=0$, which would then make transformation ( 32 ) negative-definite. We first need to establish Helmholtz ${ }^{8}$ theorem in the meridian plane. More explicitly, we want to examine the splitting of a meridian vector $\bar{P}$ into

$$
\bar{P}=g \operatorname{rad} A+\bar{t}
$$

where grad $f$, the longitudinal term, is required to be perpendicular to (c), and to have the same divergence as $\bar{P}$. In other words, $A$ must satisfy

$$
\nabla_{M}^{2} A=\operatorname{din}_{M}^{\bar{n}} \quad A=0 \text { on (c) }
$$

It is a simple matter, using relation $A 1.16$, to show that this problem has a unique solution, and that the longitudinal term vanishes when $\operatorname{div} \bar{\rho}=0$. It is also a simple matter, using Stoke's theorem in the meridian plane, to show that each meridian vector for which curl $\vec{P}=0$ can be put in the form $\operatorname{grad}_{M} \theta$. If, in addition, $\bar{P}$ is perpendicular to the boundary $c$, potential $\theta$ is nothing but the 
furction $A$ appearing in (2). The sources of $\bar{F}$ are, consequently, the curl of $\bar{f}$ and the tangertial components of $\bar{F}$.

When $f_{0}=0$, the lef hand member of (1) vanishes; this implies that the squares in the second member also varish, and, in particular, that curl $\bar{v}_{0}=0$. Letting

$$
\begin{aligned}
& \bar{v}_{0}=\operatorname{grad} A, \text { it is found that } A \text { must satisfy } \\
& \mathcal{L} \bar{v}_{0}=\nabla_{M}^{2} \text { rad } A-\frac{m^{2}}{\lambda^{2}} \operatorname{grad} A+\frac{2}{r} \bar{U}_{M} \nabla_{M} A=0 \text { wh } A=\nabla_{M}^{2} A=0 \text { on (c) }
\end{aligned}
$$

Projection of $\mathcal{L} \bar{v}_{0}^{-}$on the $\frac{7}{\partial}$ axis indicates that (u.sing AI.13)

$\frac{2}{3}\left[\nabla_{M}^{2} A-\frac{m^{2}}{2} A\right]=0$

In consequence, $\nabla_{m}^{2} A-\frac{m^{2}}{A^{2}} A$ has a constant value along a parallel to the $\bar{z}$ axis.

This value must be zero, because

$$
\nabla^{2} A-\frac{m^{2}}{r^{2}} A=0
$$

on $c$. It follows that (3) is valid over the whole area $\mathfrak{W}$. An application of Green's theorem AI. 16 shows that

$$
\log _{0}\left\{\frac{m^{2}}{a^{2}} A^{2}-|q r a d A|^{2}\right\} d r d z=0
$$

so that both $A$ and $\overline{\sqrt{s}}$ must vanish.

The self-adjoint character of $\mathscr{L}_{u}\left(\mathrm{i}_{\mathrm{o}} \mathrm{e},\langle\bar{c}, \hat{k} \bar{d}\rangle=\langle\bar{d}, \hat{h} \bar{c}\rangle\right.$ ) can be quickly established by using (AI.17) twice, setting $g=\frac{r}{m} d_{i j} \bar{P}, h=\frac{r}{m} d_{M} \bar{Q}$ and subtracting. One obtains, after using (AI. 12),

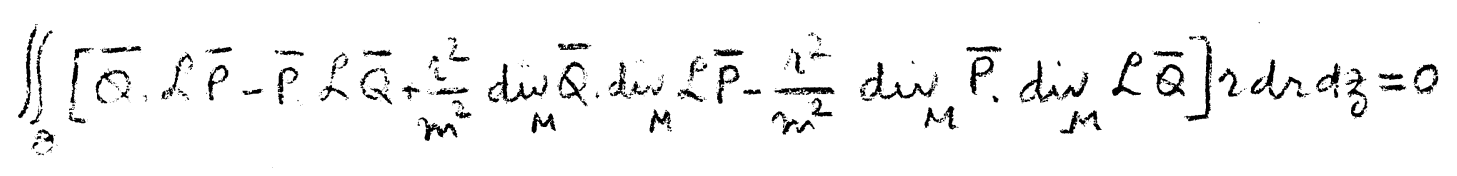

The second members in (AI. 17) vanish because of the boundary conditions. Equatior (4) is nothing but $\langle\bar{c}, k \bar{d}\rangle-\langle\bar{d} k \hat{c}\rangle=0$, the reiation we set out to prove.

Operator $\mathcal{L}$ satisfies all the conditions for its eigenvectors $\bar{c}_{\mathrm{mnp}}$ to form a closed set. And yet a paradox appears in the third equation of set (40). The 
expansion of $\bar{p}$ is rot in terms of the $\bar{c}_{m n p}$ only, but includes terms in grad $\mathcal{L}_{\text {mnp }}$ suggesting that the $\bar{c}_{\text {mnp }}$ might not form a complete set. In fact, the paradox is oniy apparent。 The terms in $\bar{c}_{m n p}$ are not the expansion of $\bar{p}_{m}$, but of its transverse part $\bar{E}$ (see equation 2). Assuming the $\bar{c}$ 's to be normalized, the expansion coefficient of the full vector would be

$$
\int_{\theta}\left[\bar{p} \cdot \bar{c}+\frac{n^{2}}{m_{m}^{2}} d w \bar{p}_{m} \cdot d_{i v \bar{c}}\right] 2 d u d z
$$

while the coefficient which actually appears in the expansion is

$$
E_{\text {ming }}=\iint_{g}\left[F_{m} \bar{c}+\frac{2}{m} U_{m} d_{m} \bar{c}\right] A d \mu d z
$$

We want to show that

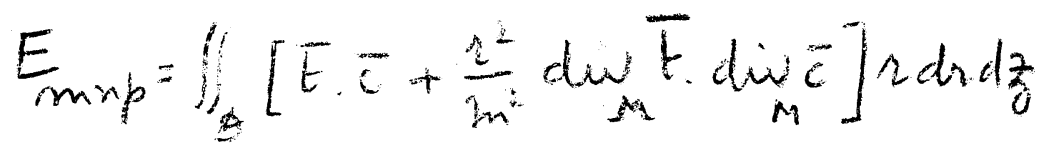

To establish the fact it is necessary to extend the content of (2). Any vector can be uniquely ${ }^{1}$ split into grad $\theta+\operatorname{curl} \vec{W}$ in a finite volume, with $\theta=0$ on the boundary surface. If we apply this Helmholtz's theorem to the vector $\left(\bar{p}_{m} \sin m+v_{m} \operatorname{com} \varphi \bar{u}_{\varphi}\right)$, we notice right away that $\theta$ and $\bar{w}$ must be of the form

$$
\begin{aligned}
& \theta=A_{m}(n, 3) \sin m \varphi+B_{m}(1,3) \operatorname{sos} m \\
& \bar{w}=\bar{\alpha}(r, z) \sin m \varphi+\bar{\beta}(r, z) \operatorname{sos} m \varphi+[c(z, z) \sin m \varphi+D(z, z) \cos r a \varphi] \bar{u}_{\varphi}
\end{aligned}
$$

It is a simple matter, by inserting these expressions in grad $\theta+\operatorname{curl} \bar{W}$, and comparing to the original vector, to check that $B_{m}=\bar{\alpha}_{m}=D_{m}=0$, and that

$$
\begin{aligned}
& \bar{P}_{m}=\operatorname{rad} A_{m}-\frac{m}{r}\left(\bar{u}_{\varphi} \times \bar{\beta}_{m}\right)+\frac{1}{r}\left[\operatorname{qrad}\left(r c_{m}\right) \times \bar{u}_{\varphi}\right] \\
& v_{m}=\frac{m A_{m}}{r}+\left|\mathrm{wad}_{M} \bar{\beta}_{m}\right|
\end{aligned}
$$

These equations can be simplified still more by noticing that curl ${ }_{M} \operatorname{grad}_{M}\left(r C_{m}\right)=0$. Setting $\bar{\gamma}=\bar{\beta}-\frac{1}{m} \operatorname{grad}\left(e_{m}\right)$, we discover that $(2)$ can be generalized into the statement that each pair $\left(\Gamma_{m}, V_{m}\right)$ splits as 
MURA-481

$$
\begin{aligned}
& \bar{p}_{m}=\operatorname{grad}_{M} A+\frac{m}{n}\left(\bar{\gamma} \times \bar{u}_{\varphi}\right) \quad \text { with } A=0 \text { on }(C) . \\
& v_{m}=\frac{m A}{n}+\mid \text { cunt }_{M_{i}} \bar{\gamma} \mid
\end{aligned}
$$

To prove the equality of $(6)$ and $(7)$, it suffices to show that

$$
\iint_{g}\left\{\left[\overline{p_{m}}-\bar{E}\right] \cdot \bar{c}+\frac{r}{m} \operatorname{din}_{M} \bar{c} \cdot\left[v_{m}-\frac{r}{m_{M}} \operatorname{din}_{M} \bar{t}\right]\right\} r d r d z=0
$$

But $\bar{P}_{m}-E=\operatorname{grad}_{M} A_{0} \operatorname{div}_{M} \bar{E}=\operatorname{miv}_{M} \frac{\bar{Y} \times \overline{\bar{u}_{4}}}{n}=\frac{m}{n}\left|\mathrm{cuml}_{M} \bar{\gamma}\right| \cdot$ Equation

(9) can consequently be rewritten as

$$
\iint_{\theta}\left\{\operatorname{qrad}_{M} A \cdot \bar{c}+\frac{r}{m} \operatorname{din}_{M} \bar{z} \cdot \frac{m A}{r}\right\} r d r d z=0
$$

This relation is a direct consequence of $A I_{0} 15$, if we remember that $A=0$ on (c).

APPENDIX III

Behavior of a continuous vector in the vicinity of the axis.

We start from Fourier expansion (1), and project on rectangular axes doxy $z$, where of is the axis of revolution. The projection on the $x$ axis yields

$$
\begin{aligned}
a_{x} & =\cos \varphi p_{02}-\sin \varphi v_{0}+\sum_{m}\left[\cos \varphi \sin m \varphi p_{m} r+\cos \varphi \cos m \varphi q_{m}\right. \\
& -\sum_{m}\left[\sin \varphi \cos m \varphi v_{m}-\sin \varphi \sin m \varphi w_{m}\right] \\
& =\frac{q_{12}+w_{1}}{2}+\cos \varphi\left(p_{02}+\frac{q_{2 r}+w_{2}}{2}\right)+\sin \varphi\left(-v_{0}+\frac{\left(p_{2}+v_{2}\right.}{2}\right) \\
& \left.+\sum_{m=2}^{\infty} \frac{\sin m \varphi\left(p_{m-1,2}-v_{m-1}+p_{m+1,2}+v_{m+1}\right)}{2}\right) \\
& +\sum_{m=2}^{\infty} \frac{\cos m \varphi}{2}\left(q_{m-1,2}-w_{m-1}+q_{m+1,2}+w_{m+1}\right)
\end{aligned}
$$

This projection has to be independent of $\varphi_{\text {when }} r=0$. A11 coefficients of the $\sin \varphi, \cos \varphi, \sin m \varphi$ and $\cos m \varphi$ terms must consequently vanish. This yields the conditions:

$$
-v_{0}+\frac{p_{2}}{2}+\frac{v_{2}}{2}=0
$$

36 
MURA -481

$$
\begin{aligned}
& p_{m 2}+\frac{q_{22}}{2}+\frac{w_{2}}{2}=0 \\
& p_{m+12}+p_{m+1 / 2}-v_{m-1}+v_{m+1}=0 \\
& q_{m-1,2}+q_{m+1}-w_{m+1}+w_{m+1}=0 \text { for } m \geq 2
\end{aligned}
$$

Similarly, ky projecting on the $y$ axis, ore obtains the conditions

$$
\begin{aligned}
& v_{0}+\frac{p_{2}}{2}+\frac{v_{2}}{2}=0 \\
& p_{m 2}-\frac{q_{2}}{2}-\frac{w_{2}}{2}=0 \\
& -p_{m-1,}+p_{m+1,2}+v_{m-1}+v_{m+1}=0 \quad \text { fm } m \geq 2 \\
& q_{m+2}+q_{m+1,2}+w_{m-1}+w
\end{aligned}
$$

Projection on the $z$ axis indicates simply that $p_{m z}$ and $q_{m z}$ must vanish, while $f_{0 z}$ can have any value. The conditions mentioned in the text can now easily be derived by considering relations (2) and (3) together 。 The first relations, for example, yield immediately $v_{0}=0$ and $p_{2 r}+v_{2}=0$ 。 The third relations, written as

$$
\begin{aligned}
& p_{22}-v_{2}+p_{4 n}+v_{4}=0 \\
& -p_{2 \Omega}-v_{2}+p_{4 n}+v_{4}=0 \quad\left(k^{2} m=3\right)
\end{aligned}
$$

indicate that $p_{2 r} \times v_{2}=0$. As a result, $v_{2}=0$ and $p_{2 r}=0$ 。

37 